\title{
Pattern Formation in a Three-Species Cyclic Competition Model
}

\author{
Kalyan Manna ${ }^{1} \cdot$ Vitaly Volpert $^{2,3,4} \cdot$ Malay Banerjee ${ }^{1}$ (D
}

Received: 21 August 2020 / Accepted: 9 March 2021

(c) The Author(s), under exclusive licence to Society for Mathematical Biology 2021

\begin{abstract}
In nature, different species compete among themselves for common resources and favorable habitat. Therefore, it becomes really important to determine the key factors in maintaining the bio-diversity. Also, some competing species follow cyclic competition in real world where the competitive dominance is characterized by a cyclic ordering. In this paper, we study the formation of a wide variety of spatiotemporal patterns including stationary, periodic, quasi-periodic and chaotic population distributions for a diffusive Lotka-Volterra type three-species cyclic competition model with two different types of cyclic ordering. For both types of cyclic ordering, the temporal dynamics of the corresponding non-spatial system show the extinction of two species through global bifurcations such as homoclinic and heteroclinic bifurcations. For the spatial system, we show that the existence of Turing patterns is possible for a particular cyclic ordering, while it is not the case for the other cyclic ordering through both the analytical and numerical methods. Further, we illustrate an interesting scenario of short-range invasion as opposed to the usual invasion phenomenon over the entire habitat. Also, our study reveals that both the stationary and dynamic population distributions can coexist in different parts of a habitat. Finally, we extend the spatial system by incorporating nonlocal intra-specific competition terms for all the three competing species. Our study shows that the introduction of nonlocality in intra-specific competitions stabilizes the system dynamics by transforming a dynamic population distribution to stationary. Surprisingly, this nonlocality-induced stationary pattern formation leads to the extinction of one species and hence, gives rise to the loss of bio-diversity for intermediate ranges of nonlocality. However, the bio-diversity can be restored for sufficiently large extent of nonlocality.
\end{abstract}

Keywords Cyclic competition model $\cdot$ Diffusion $\cdot$ Nonlocal interactions $\cdot$ Hopf bifurcation · Turing instability $\cdot$ Pattern formation 


\section{Introduction}

In real world, different species can sometimes compete among themselves in a nontransitive manner where the competitive dominance follows a cyclic ordering, and accordingly, this type of competition is called a cyclic competition (Adamson and Morozov 2012; Moen 1989). Some of the well-documented examples of cyclic competition in real world include the competition among several coral reef invertebrate species (Buss and Jackson 1979; Jackson and Buss 1975), few strains of yeast (Paquin and Adams 1983) and certain bacteria strains (Kerr et al. 2002; Kirkup and Riley 2004). In the literature, it is reported that non-spatial cyclic competition models typically predict the loss of bio-diversity through the extinction of one or more competing species. For example, May and Leonard (May and Leonard 1975) investigated the temporal dynamics of a three-species Lotka-Volterra type symmetric cyclic competition model and suggested the extinction of two competing species. Some preliminary results were reported in Gilpin (1975) where oscillatory coexistence was found in ecological communities with an odd number ( $\geq 3$ ) of competing species. Coste et al. (1979) showed that periodic attractors arising through the Hopf bifurcation can exist for a small range of parameter values. Further, Schuster et al. (1979) studied some competition models for three species which exhibit the survival of one species depending upon the initial population density. Chaotic oscillation, non-existence of oscillatory coexistence and competition for different resources were studied in Baer et al. (2006), Chi et al. (1998), Gardini et al. (1989).

There exists a large body of studies on spatiotemporal dynamics of two- and multidimensional prey-predator systems; however, only a few studies have been conducted for spatially extended competition models (especially, for cyclic competition models with three or more species). It is well-known that the Lotka-Volterra type two-species competition model with only self-diffusion does not admit any stationary pattern (Han et al. 2011; Lou et al. 2000; Ni et al. 2018). Nevertheless, the inclusion of crossdiffusion in the modeling approach can effectively produce stationary patches for two-species competition models (Li et al. 2019; Lou and Ni 1996). Kishimoto showed that a stable non-constant equilibrium solution can exist for the diffusive three-species Lotka-Volterra type competition model in Kishimoto (1982). Merino (1996) considered a diffusive cyclic competition model of three species and proved the existence of a temporally periodic solution. Lou et al. (2000) asserted the cross-diffusion mediated existence of heterogeneous patches in a three-species Lotka-Volterra type competition model. By investigating the dynamics of a diffusive cyclic competition model for three species, the authors showed the existence of both the traveling waves and spatiotemporal chaos in Petrovskii et al. (2001). Adamson and Morozov (2012) revisited the cyclic competition model considered in Petrovskii et al. (2001) to explore the role of species dispersal in maintaining bio-diversity. It should be noted that there does not exist any study in the literature which explicitly explores the stationary pattern formation in cyclic competition models of three species as far as our knowledge goes.

The above-mentioned studies on spatially extended competition models assume both the intra- and inter-specific competitions as local competitions which allow an individual located at some spatial point to interact with other individuals (from the same or different species) present at that point. However, spatial mobility of a species leads to 
the resource depletion in a neighboring region around that specific point (Britton 1989). Therefore, both the intra- and inter-specific competitions for the common resources should be controlled by an average population density in that neighborhood instead of being local, and accordingly, this type of competitions are called nonlocal competitions (Autry et al. 2018; Banerjee and Volpert 2016; Britton 1989; Tanzy et al. 2013). The nonlocal competitions are incorporated in the modeling approach through convolution integrals with a specific kernel function and hence, a system of integro-differential equations arise (Autry et al. 2018; Banerjee and Volpert 2016; Britton 1989; Tanzy et al. 2013). Examples of kernel function include top-hat, triangular, parabolic, Gaussian and Laplacian kernels (Manna et al. 2020; Merchant and Nagata 2011; Pal et al. 2019; Segal et al. 2013).

A considerable number of studies on prey-predator systems with spatial kernels to model nonlocal interaction have been conducted over the last decade and some interesting works can be found in Autry et al. (2018), Banerjee and Volpert (2016), Bayliss and Volpert (2017), Manna et al. (2020), Merchant and Nagata (2011), Merchant and Nagata (2015), Pal et al. (2018), Pal et al. (2019). However, a very limited number of works on nonlocal competition models can be found in the literature. Apreutesei et al. (2008) showed the existence of traveling waves for short range of nonlocal interactions by using Fredholm property and solvability conditions of the linear integro-differential operators. Also, they observed the propagation of periodic waves instead of the usual traveling waves for sufficiently large support of the kernel. Segal et al. (2013) investigated the effects of three different kernels on the spatiotemporal dynamics of a nonlocal two-species competition model, and revealed that the population distributions form islands (or localized cells) for top-hat kernel, whereas the consideration of parabolic kernel can produce more complex patterns by modulating the amplitude of islands and localized extinction. Also, they demonstrated the tip-splitting phenomenon within islands for triangular kernel. Similarly, a nonlocal two-species competition model with two different kernel functions such as asymmetric Gaussian and asymmetric step-function kernels was studied in Tanzy et al. (2013). This study revealed a range of patterns including dynamic patches with fixed and variable widths. Ni et al. (2018) reported stationary population patches for a spatial two-species competition model with the nonlocal intra- and inter-specific competitions.

In this paper, we consider a generalized version of the three-species cyclic competition model previously studied in Adamson and Morozov (2012), Cangiani et al. (2018), Petrovskii et al. (2001) and investigate its dynamics without and with nonlocal intra-specific competitions. Also, we are interested to explore the stabilizing effects of nonlocal competitions on the emerging spatiotemporal dynamics. We organize the rest of this article in the following manner. In the next section, we introduce the spatial cyclic competition model for three competing species and present a detailed study on the resulting spatiotemporal dynamics via both the analytical and numerical tools. In order to clarify the mechanisms behind the emerging spatiotemporal dynamics, we encapsulate the dynamics possessed by the corresponding non-spatial system in Sect. 2.1. Further, we extend the spatial system by considering nonlocal intra-specific competitions for all the three competing species and investigate the effects of nonlo- 
cality on the resulting spatiotemporal dynamics in Sect. 3. Finally, Sect. 4 concludes this study with a brief discussion and ecological interpretations of the obtained results.

\section{Local Reaction-Diffusion System}

In this section, we consider a spatially extended three species cyclic competition model and investigate its spatiotemporal dynamics. The spatiotemporal dynamics for the model with specific form of kinetic parameters have been investigated in Adamson and Morozov (2012), Cangiani et al. (2018), Petrovskii et al. (2001). Here, we wish to investigate the dynamics of the model with more general setting in terms of the kinetic parameters. For simplicity, we consider one-dimensional bounded spatial domain $[-L, L]$ with positive real number $L$. Accordingly, our spatial model of interest is given by:

$$
\begin{aligned}
& \frac{\partial N_{1}}{\partial t}=d_{1} \frac{\partial^{2} N_{1}}{\partial x^{2}}+\left(r_{1}-a_{11} N_{1}-a_{12} N_{2}-a_{13} N_{3}\right) N_{1}, \\
& \frac{\partial N_{2}}{\partial t}=d_{2} \frac{\partial^{2} N_{2}}{\partial x^{2}}+\left(r_{2}-a_{21} N_{1}-a_{22} N_{2}-a_{23} N_{3}\right) N_{2}, \\
& \frac{\partial N_{3}}{\partial t}=d_{3} \frac{\partial^{2} N_{3}}{\partial x^{2}}+\left(r_{3}-a_{31} N_{1}-a_{32} N_{2}-a_{33} N_{3}\right) N_{3},
\end{aligned}
$$

subjected to non-negative initial conditions and periodic boundary conditions. Here, $N_{j}(x, t)$ with $j=1,2,3$ represent the density of species $j$ at time $t$ and spatial position $x$. The parameters $r_{j}$ and $d_{j}$ account for the intrinsic growth rate and diffusion coefficient, respectively, for species $j$. Coefficients $a_{j s}$ denote intra-specific (when $j=$ $s$ ) and inter-specific (when $j \neq s$ ) competition rates, respectively, with $j, s=1,2,3$. From the ecological perspective, we consider all the parameters involved in system (1-3) to be positive.

The above system (1-3) presents a classical Lotka-Volterra type competition model for three species (May and Leonard 1975). Before proceeding further, it would be appropriate to define the feature which actually makes the system (1-3) a cyclic competition model. Basically, cyclic competition defines a well-ordered competitive dominance of one species over the other (Adamson and Morozov 2012). Such competitive advantages ordered in a cycle can be efficiently incorporated in the modeling approach by putting appropriate restrictions on inter-specific competition rates $a_{j s}$ $(j \neq s)$. For example, the restrictions $a_{12}<a_{21}, a_{23}<a_{32}$ and $a_{31}<a_{13}$ imposed in a three-species competition system signify the cyclic competition where species 1 outcompetes species 2, species 2 outcompetes species 3 and species 3 outcompetes species 1. Similarly, if we flip the inequalities in the above restrictions (that is, $a_{12}>a_{21}, a_{23}>a_{32}$ and $\left.a_{31}>a_{13}\right)$ then we end up with a cyclic competition in reverse order where species 1 outcompetes species 3 , species 3 outcompetes species 2 and species 2 outcompetes species 1 . We are particularly interested in this specific ordering of the cyclic competition as it can induce the Turing instability (see Sect. 2.2). For a detailed discussion on cyclic competition, one can go through the 
references (Adamson and Morozov 2012; Cangiani et al. 2018). These are only two specific ordering for cyclic competition and there exist several other examples of three-species competition models depending on the relationships among inter-specific competition rates. However, we do not consider those models in this study.

Now, we discuss the existence of possible spatially homogeneous steady states of the system (1-3). A typical spatially homogeneous steady state $E=\left(\widetilde{N_{1}}, \widetilde{N_{2}}, \widetilde{N_{3}}\right)$ of the system (1-3) corresponds to a non-negative solution of the following system of coupled algebraic equations:

$$
\begin{aligned}
& \left(r_{1}-a_{11} \widetilde{N}_{1}-a_{12} \widetilde{N_{2}}-a_{13} \widetilde{N_{3}}\right) \widetilde{N_{1}}=0,\left(r_{2}-a_{21} \widetilde{N}_{1}-a_{22} \widetilde{N}_{2}-a_{23} \widetilde{N}_{3}\right) \widetilde{N_{2}}=0 \\
& \left(r_{3}-a_{31} \widetilde{N}_{1}-a_{32} \widetilde{N}_{2}-a_{33} \widetilde{N}_{3}\right) \widetilde{N}_{3}=0 .
\end{aligned}
$$

Solving the above system of algebraic equations, we obtain the following spatially homogeneous steady states for the system (1-3):

(i) Total extinction steady state $E_{0} \equiv(0,0,0)$. Note that $E_{0}$ exists always.

(ii) Three one-species steady states $E_{+00} \equiv\left(\frac{r_{1}}{a_{11}}, 0,0\right), E_{0+0} \equiv\left(0, \frac{r_{2}}{a_{22}}, 0\right)$ and $E_{00+} \equiv\left(0,0, \frac{r_{3}}{a_{33}}\right)$. As all the kinetic parameters are positive, these three onespecies steady states also exist for all possible combinations of feasible parameter values.

(iii) Three two-species steady states $E_{++0} \equiv\left(\frac{\zeta_{12}}{\rho_{12}}, \frac{\zeta_{21}}{\rho_{12}}, 0\right), E_{0++} \equiv\left(0, \frac{\zeta_{23}}{\rho_{23}}, \frac{\zeta_{32}}{\rho_{23}}\right)$ and $E_{+0+} \equiv\left(\frac{\zeta_{13}}{\rho_{13}}, 0, \frac{\zeta 31}{\rho_{13}}\right)$, where $\zeta_{j s}=r_{j} a_{s s}-r_{s} a_{j s}$ (for $j, s \in\{1,2,3\}$ with $j \neq s$ ) and $\rho_{j s}=a_{j j} a_{s s}-a_{j s} a_{s j}$ (for $j=1,2 ; s=2,3$; and $j<s$ ). Each of these two-species steady states exists provided both the corresponding non-zero components assume positive values.

(iv) Unique coexistence steady state $E_{*} \equiv\left(N_{1}^{*}, N_{2}^{*}, N_{3}^{*}\right)$, where the components are given by

$$
\begin{aligned}
N_{1}^{*} & =\frac{\zeta_{13} \rho_{23}-\zeta_{23}\left(a_{12} a_{33}-a_{13} a_{32}\right)}{\rho_{13} \rho_{23}-\left(a_{12} a_{33}-a_{13} a_{32}\right)\left(a_{21} a_{33}-a_{23} a_{31}\right)}, \\
N_{2}^{*} & =\frac{\zeta_{23} \rho_{13}-\zeta_{13}\left(a_{21} a_{33}-a_{23} a_{31}\right)}{\rho_{13} \rho_{23}-\left(a_{12} a_{33}-a_{13} a_{32}\right)\left(a_{21} a_{33}-a_{23} a_{31}\right)}, \\
N_{3}^{*} & =\frac{1}{a_{33}}\left(r_{3}-a_{31} N_{1}^{*}-a_{32} N_{2}^{*}\right) .
\end{aligned}
$$

The coexistence steady state is feasible in a parametric regime where each component $N_{j}^{*}(j=1,2,3)$ assumes positive values.

In this study, we are mainly interested in the spatiotemporal dynamics possessed by the system (1-3) around the unique spatially homogeneous coexistence steady state $E_{*}$ as this state accounts for the bio-diversity. 


\subsection{Dynamics of the Non-spatial System: A Brief Summary}

The consideration that all the three competing species are distributed uniformly in their natural habitat essentially leads to a non-spatial system. It is believed that the conclusions drawn from a non-spatial system work well for the well-mixed populations. Generally, modeling through non-spatial system leaves out many realistic features (such as self-movement of populations) of natural ecosystems, and hence, conclusions drawn from their study may be far from reality. However, prior knowledge about the temporal dynamics plays a significant role in understanding the mechanisms of the corresponding spatiotemporal dynamics adequately. Thus, we briefly discuss the dynamics of the non-spatial system corresponding to the spatial system (1-3) in this subsection. The corresponding non-spatial system is given by

$$
\begin{aligned}
& \frac{d N_{1}}{d t}=\left(r_{1}-a_{11} N_{1}-a_{12} N_{2}-a_{13} N_{3}\right) N_{1}, \\
& \frac{d N_{2}}{d t}=\left(r_{2}-a_{21} N_{1}-a_{22} N_{2}-a_{23} N_{3}\right) N_{2}, \\
& \frac{d N_{3}}{d t}=\left(r_{3}-a_{31} N_{1}-a_{32} N_{2}-a_{33} N_{3}\right) N_{3},
\end{aligned}
$$

with non-negative initial conditions. Note that the steady states for this non-spatial system (4-6) are actually the spatially homogeneous steady states of the spatial system (1-3), and accordingly, we use the same notations to denote them. As the coexistence steady state $E_{*}$ accounts for the bio-diversity in an ecological system, we wish to examine the dynamical behaviors of the system around $E_{*}$. We would like to mention here that May and Leonard (May and Leonard 1975) thoroughly investigated the temporal dynamics of the non-spatial system (4-6) with some specific restrictions on the system parameters. Some other theoretical studies on this three species competition model can be found in Chi et al. (1998), Coste et al. (1979), Schuster et al. (1979), Smale (1976). Here, we wish to present results regarding the linear stability of $E_{*}$ and Hopf bifurcation about $E_{*}$ by maintaining the generality of the system parameters and in turn, it would effectively capture some additional dynamics in larger parametric regimes which are beyond the scope of the study presented in May and Leonard (1975).

Now, linearizing the above system (4-6) about the coexistence steady state $E_{*}=$ $\left(N_{1}^{*}, N_{2}^{*}, N_{3}^{*}\right)$, we obtain the following characteristic equation

$$
\lambda^{3}+A \lambda^{2}+B \lambda+C=0,
$$

where

$$
\begin{aligned}
A= & a_{11} N_{1}^{*}+a_{22} N_{2}^{*}+a_{33} N_{3}^{*}, \\
B= & \rho_{12} N_{1}^{*} N_{2}^{*}+\rho_{23} N_{2}^{*} N_{3}^{*}+\rho_{13} N_{1}^{*} N_{3}^{*}, \\
C= & \left(a_{11} a_{22} a_{33}+a_{12} a_{23} a_{31}+a_{13} a_{21} a_{32}-a_{11} a_{23} a_{32}-a_{12} a_{21} a_{33}\right. \\
& \left.-a_{13} a_{22} a_{31}\right) N_{1}^{*} N_{2}^{*} N_{3}^{*} .
\end{aligned}
$$


Then, the coexistence steady state $E_{*}$ is locally asymptotically stable if the following Routh-Hurwitz criteria are satisfied:

$$
A>0, C>0 \text { and } A B-C>0 .
$$

We can easily observe that the first inequality $A>0$ in (8) is immediately satisfied for a feasible coexistence steady state $E_{*}$. In order to satisfy the second inequality $C>0$, we need to consider a parametric restriction

$$
a_{11} a_{22} a_{33}+a_{12} a_{23} a_{31}+a_{13} a_{21} a_{32}>a_{11} a_{23} a_{32}+a_{12} a_{21} a_{33}+a_{13} a_{22} a_{31},
$$

which acts as a necessary condition for local stability of $E_{*}$. Also, the last inequality in (8) implies

$$
\begin{aligned}
& \left(a_{11} N_{1}^{*}+a_{22} N_{2}^{*}\right) \rho_{12} N_{1}^{*} N_{2}^{*}+\left(a_{22} N_{2}^{*}+a_{33} N_{3}^{*}\right) \rho_{23} N_{2}^{*} N_{3}^{*}+ \\
& \left(a_{11} N_{1}^{*}+a_{33} N_{3}^{*}\right) \rho_{13} N_{1}^{*} N_{3}^{*}+\left(a_{33} \rho_{12}+a_{22} \rho_{13}\right) N_{1}^{*} N_{2}^{*} N_{3}^{*}>0 .
\end{aligned}
$$

Now, a Hopf bifurcation occurs around $E_{*}$ when a pair of complex conjugate eigenvalues pass through the imaginary axis while the third eigenvalue remains to be a negative real number. Let us assume that $\lambda_{1}, \lambda_{2}$ and $\lambda_{3}$ denote the three eigenvalues corresponding to the characteristic Eq. (7). Then, from the properties of the roots of a cubic equation, we obtain

$$
-\left(\lambda_{1}+\lambda_{2}\right)\left(\lambda_{2}+\lambda_{3}\right)\left(\lambda_{3}+\lambda_{1}\right)=A B-C .
$$

If we consider $a_{13}$ as the bifurcation parameter, then at the Hopf bifurcation threshold $a_{13}=a_{13}^{*}$ we have $A B-C=0$. However, we need to satisfy the transversality condition in order to guarantee the occurrence of Hopf bifurcation. Therefore, the non-spatial system (4-6) undergoes Hopf bifurcation at $a_{13}=a_{13}^{*}$ if the following conditions hold:

$$
\left.(A B-C)\right|_{a_{13}=a_{13}^{*}}=0,\left.\quad \frac{d}{d a_{13}}(A B-C)\right|_{a_{13}=a_{13}^{*}} \neq 0,
$$

along with the parametric restriction

$$
a_{11} a_{22} a_{33}+a_{12} a_{23} a_{31}+a_{13}^{*} a_{21} a_{32}>a_{11} a_{23} a_{32}+a_{12} a_{21} a_{33}+a_{13}^{*} a_{22} a_{31}
$$

which arises due to the condition $\left.C\right|_{a_{13}=a_{13}^{*}}>0$. Further, the stability of the Hopfbifurcating periodic solutions can be easily determined from the sign of the first Lyapunov coefficient (say, $\gamma$ ). For smooth readability, we do not include the rigorous derivation of this quantity $\gamma$ here. The detailed derivation of the quantity $\gamma$ has been provided in the Supplementary material.

Now, we present some numerical simulations of the non-spatial system (4-6) which illustrate our theoretical findings and some other dynamical features that are beyond 
the scope of our theoretical analysis. For this purpose, we considered the following set of parameter values:

$$
\begin{aligned}
& r_{1}=2, r_{2}=2, r_{3}=2, a_{11}=0.8, a_{22}=1.2, a_{33}=0.8, a_{12}=1.2, a_{21}=0.7 \\
& a_{23}=1.2, a_{31}=1.2, a_{32}=0.7
\end{aligned}
$$

and treated the rate of competition pressure exerted by the third species on the first species (that is, $a_{13}$ ) as the bifurcation parameter. An increase in the competition pressure leads to the oscillatory coexistence of all the competing species by destroying the stable steady state coexistence (see Fig. 1a and b). The destabilization takes place through a super-critical Hopf bifurcation (the bifurcation threshold is mentioned in the caption of Fig. 1 and the quantity $\gamma$ is negative in this case). However, the oscillatory coexistence is observed for a narrow range of the values of $a_{13}$. Further increment in the value of $a_{13}$ leads to the disappearance of stable population cycle through the homoclinic bifurcation and only the third species survives at its carrying capacity (see Fig. 1c and d). This phenomenon arises due to the fact that the third species becomes a superior competitor in this case and it eventually drives the first two species to extinction by outcompeting them.

At this stage, we would like to illustrate the temporal dynamics of the three species competition model studied in May and Leonard (1975) which represents a specific version of our considered general non-spatial system (4-6). Taking $r_{1}=r_{2}=r_{3}=1$, $a_{11}=a_{22}=a_{33}=1, a_{12}=a_{23}=a_{31}=\alpha$ and $a_{13}=a_{21}=a_{32}=\beta$ in our system (4-6), we obtain the model presented in May and Leonard (1975). For these specific parametrization, the steady states are given by $E_{0}=(0,0,0), E_{+00}=$ $(1,0,0), E_{0+0}=(0,1,0), E_{00+}=(0,0,1), E_{++0}=\left(\frac{1-\alpha}{1-\alpha \beta}, \frac{1-\beta}{1-\alpha \beta}, 0\right), E_{0++}=$ $\left(0, \frac{1-\alpha}{1-\alpha \beta}, \frac{1-\beta}{1-\alpha \beta}\right), E_{+0+}=\left(\frac{1-\beta}{1-\alpha \beta}, 0, \frac{1-\alpha}{1-\alpha \beta}\right)$ and $E_{*}=\left(\frac{1}{1+\alpha+\beta}, \frac{1}{1+\alpha+\beta}, \frac{1}{1+\alpha+\beta}\right)$. The local stability of the coexistence steady state $E_{*}$ for this specific model is guaranteed by the inequality $\alpha+\beta<2$ with $\alpha, \beta>0$. For $\alpha+\beta \geq 2$, all the feasible steady states are unstable when $\alpha<1$ or $\beta<1$. Further, any one of the single-species steady states becomes stable when both the $\alpha$ and $\beta$ are greater than unity. Note that a pair of complex conjugate eigenvalues for the system linearized about $E_{*}$ moves from the left half of the complex plane to the right half through $\alpha+\beta=2$. The detailed stability analysis in this direction can be found in May and Leonard (1975).

Some of the numerical simulations for this specific model are encapsulated in Fig. 2. For the simulations, we used $\alpha=0.8<1$ and varied the parameter $\beta$. In this case, the stable steady state coexistence is destroyed and a stable oscillatory coexistence appears for an appropriate increased value of $\beta$ (see Fig. $2 \mathrm{a}$ and b). The stable limit cycle disappears through the heteroclinic bifurcation and a heteroclinic cycle appears connecting all the three single-species steady states for further increment in the value of $\beta$ (see Fig. 2c). After its appearance, the heteroclinic cycle persists for all greater values of $\beta$. However, the heteroclinic cycles consist of curved orbits for sufficiently large values of $\beta$ (see Fig. 2d). Hopf-bifurcating limit cycles disappear through global bifurcations like homoclinic and heteroclinic bifurcations and it leads to the system collapse due to the extinction of one or more species (van Voorn et al. 2007). In 


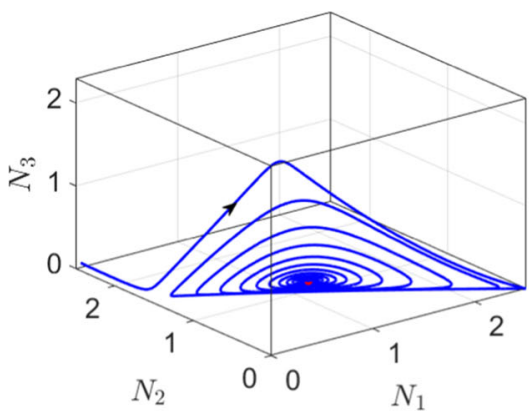

(a)

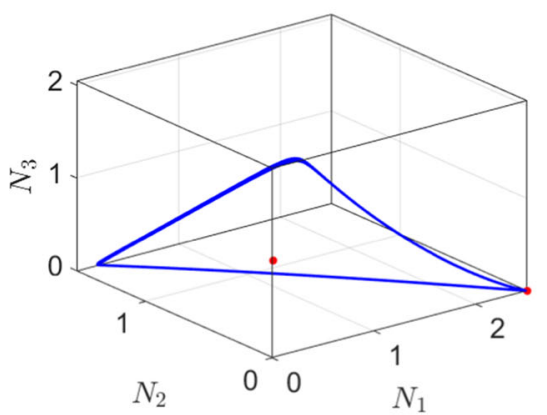

(c)

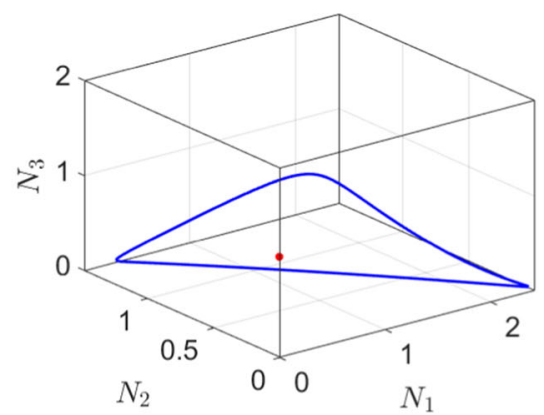

(b)

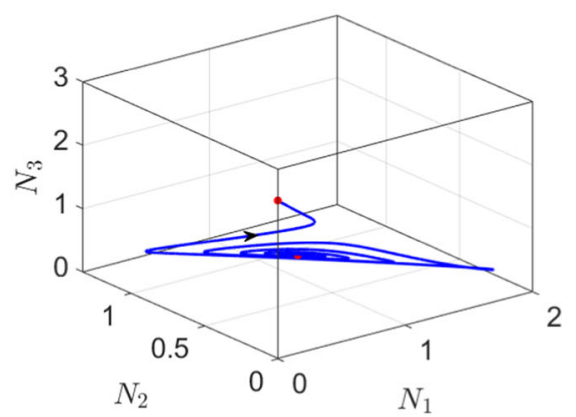

(d)

Fig. 1 Phase portraits of the non-spatial system (4-6) for different values of the coefficient $a_{13}$ accounting for the competition pressure of species 3 on species 1 . The plots are for $\mathbf{a} a_{13}=0.7, \mathbf{b} a_{13}=0.8002$, c $a_{13}=0.807$, and (d) $a_{13}=0.9$. Other parameter values are mentioned in (11). The Hopf and homoclinic bifurcation thresholds are $a_{13}^{*}=0.8$ and $a_{13}^{\mathrm{HM}}=0.807$, respectively (Color Figure Online)

this case, the heteroclinic cycle persists beyond the onset of such cycle and this is a remarkable characteristic for the three-species competition model. The existence of a stable limit cycle demonstrates the oscillatory coexistence of all the three competitive species. However, the existence of heteroclinic population cycle indicates the system collapse where only one species survives at its carrying capacity depending upon the initial population density. Some of the figures (specifically, Fig. 2b and c) presented here have been illustrated schematically in May and Leonard (1975). The persistence of heteroclinic orbit for $\beta \geq 1.205$ is a new observation of our present work. Overall, the simulation results presented in Fig. 2 capture the corresponding temporal dynamics in a more complete manner.

\subsection{Dynamics of the Spatial System: Turing Instability}

In this subsection, we investigate the dynamics of the spatial system (1-3). In this direction, we mainly concentrate on the diffusion-driven or Turing instability of the spatially homogeneous coexistence steady state $E_{*}$ through linear analysis. Turing instability occurs when the locally stable spatially uniform state $E_{*}$ (in the absence 


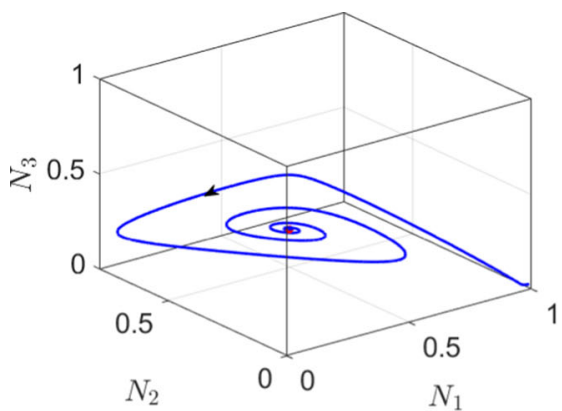

(a)

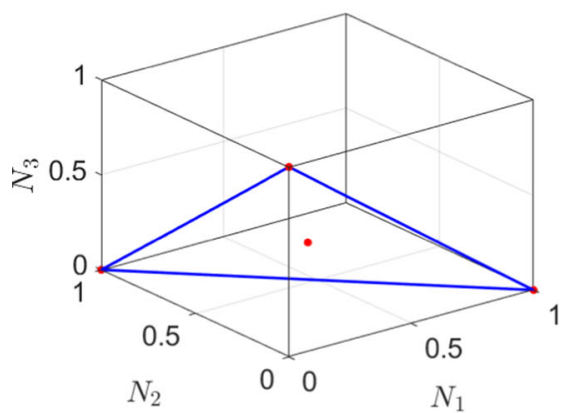

(c)

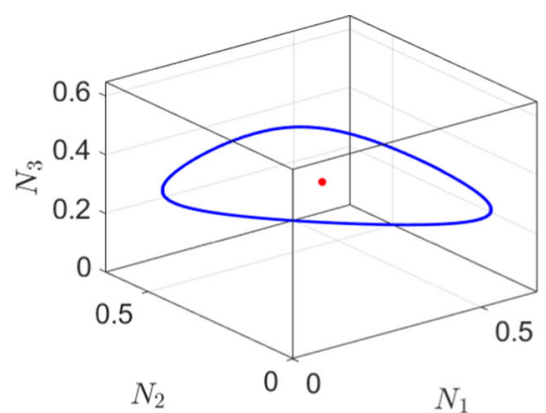

(b)

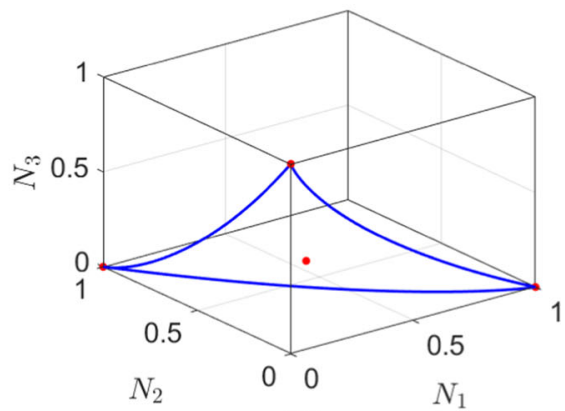

(d)

Fig. 2 Phase portraits of the specific version of our non-spatial system (4-6) studied in May and Leonard (1975) with $\alpha=0.8$ and different values of $\beta$ : $\mathbf{a} \beta=1.1, \mathbf{b} \beta=1.2, \mathbf{c} \beta=1.205$, and $\mathbf{d} \beta=1.9$ (Color Figure Online)

of diffusion) becomes unstable to nonuniform perturbations precisely due to diffusion (Murray 1989; Turing 1952). Basically, the presence of diffusion makes the nonuniform perturbations growing and eventually a spatially heterogeneous structure in population distribution emerges (Murray 1989; Turing 1952). For this purpose, we perturb the system (1-3) with small amplitude spatiotemporal perturbation about the spatially homogeneous coexistence steady state $E_{*}=\left(N_{1}^{*}, N_{2}^{*}, N_{3}^{*}\right)$ and then linearize the system. The corresponding Jacobian matrix is given by

$$
\mathcal{J}(k)=\left[\begin{array}{ccc}
-a_{11} N_{1}^{*}-d_{1} k^{2} & -a_{12} N_{1}^{*} & -a_{13} N_{1}^{*} \\
-a_{21} N_{2}^{*} & -a_{22} N_{2}^{*}-d_{2} k^{2} & -a_{23} N_{2}^{*} \\
-a_{31} N_{3}^{*} & -a_{32} N_{3}^{*} & -a_{33} N_{3}^{*}-d_{3} k^{2}
\end{array}\right],
$$

where the wavenumber is denoted by $k$. Then, we obtain the following characteristic equation

$$
\lambda^{3}+A\left(k^{2}\right) \lambda^{2}+B\left(k^{2}\right) \lambda+C\left(k^{2}\right)=0,
$$


where

$$
\begin{aligned}
A\left(k^{2}\right)= & \left(d_{1}+d_{2}+d_{3}\right) k^{2}+a_{11} N_{1}^{*}+a_{22} N_{2}^{*}+a_{33} N_{3}^{*}, \\
B\left(k^{2}\right)= & \left(d_{1} d_{2}+d_{2} d_{3}+d_{3} d_{1}\right) k^{4}+\left\{\left(a_{22} N_{2}^{*}+a_{33} N_{3}^{*}\right) d_{1}+\left(a_{11} N_{1}^{*}+a_{33} N_{3}^{*}\right) d_{2}\right. \\
& \left.+\left(a_{11} N_{1}^{*}+a_{22} N_{2}^{*}\right) d_{3}\right\} k^{2}+\rho_{12} N_{1}^{*} N_{2}^{*}+\rho_{23} N_{2}^{*} N_{3}^{*}+\rho_{13} N_{1}^{*} N_{3}^{*}, \\
C\left(k^{2}\right)= & d_{1} d_{2} d_{3} k^{6}+\left(a_{11} N_{1}^{*} d_{2} d_{3}+a_{22} N_{2}^{*} d_{1} d_{3}+a_{33} N_{3}^{*} d_{1} d_{2}\right) k^{4}+\left\{\rho_{12} N_{1}^{*} N_{2}^{*} d_{3}\right. \\
& \left.+\rho_{23} N_{2}^{*} N_{3}^{*} d_{1}+\rho_{13} N_{1}^{*} N_{3}^{*} d_{2}\right\} k^{2}+\left(a_{11} a_{22} a_{33}+a_{12} a_{23} a_{31}+a_{13} a_{21} a_{32}\right. \\
& \left.-a_{11} a_{23} a_{32}-a_{12} a_{21} a_{33}-a_{13} a_{22} a_{31}\right) N_{1}^{*} N_{2}^{*} N_{3}^{*} .
\end{aligned}
$$

Therefore, the spatially homogeneous coexistence steady state $E_{*}$ is locally asymptotically stable provided the following Routh-Hurwitz criteria

$$
A\left(k^{2}\right)>0, C\left(k^{2}\right)>0 \text { and } A\left(k^{2}\right) B\left(k^{2}\right)-C\left(k^{2}\right)>0
$$

are satisfied for all $k>0$. In this case, real parts of all the eigenvalues are negative. On the other hand, Turing instability emerges when the real parts of two eigenvalues remain negative and the remaining one eigenvalue passes through zero. Without any loss of generality, we assume that $\lambda_{1}, \lambda_{2}$ and $\lambda_{3}$ represent the roots of the characteristic Eq. (13), and accordingly, we obtain

$$
\begin{aligned}
\lambda_{1}+\lambda_{2}+\lambda_{3} & =-A\left(k^{2}\right), \\
\lambda_{1} \lambda_{2}+\lambda_{2} \lambda_{3}+\lambda_{3} \lambda_{1} & =B\left(k^{2}\right), \\
\lambda_{1} \lambda_{2} \lambda_{3} & =-C\left(k^{2}\right), \\
-\left(\lambda_{1}+\lambda_{2}\right)\left(\lambda_{2}+\lambda_{3}\right)\left(\lambda_{3}+\lambda_{1}\right) & =A\left(k^{2}\right) B\left(k^{2}\right)-C\left(k^{2}\right) .
\end{aligned}
$$

Let us assume that $k_{T}$ denote the critical wavenumber at the Turing bifurcation threshold. Thus, at this critical wavenumber $k=k_{T}$ we have without any loss of generality

$$
\left.\lambda_{1}\right|_{k^{2}=k_{T}^{2}}=0,\left.\operatorname{Re}\left(\lambda_{2}\right)\right|_{k^{2}=k_{T}^{2}}<0 \text { and }\left.\operatorname{Re}\left(\lambda_{3}\right)\right|_{k^{2}=k_{T}^{2}}<0 .
$$

Hence, we have $C\left(k^{2}\right)=0$ at the critical wavenumber $k=k_{T}$. Further, the conditions for Turing instability presented in (19) leads to $A\left(k_{T}^{2}\right)>0, B\left(k_{T}^{2}\right)>0$ and $A\left(k_{T}^{2}\right) B\left(k_{T}^{2}\right)-C\left(k_{T}^{2}\right)=A\left(k_{T}^{2}\right) B\left(k_{T}^{2}\right)>0$. Thus, the coexistence steady state $E_{*}$ becomes Turing unstable when $C\left(k^{2}\right)<0$ holds for at least one $k>0$ and it remains stable when $C\left(k^{2}\right)>0$ holds for all $k \geq 0$. Also, beyond the Turing bifurcation threshold there exists a range of $k$-values around $k_{T}$ for which the inequality $C\left(k^{2}\right)<0$ holds true. We can rewrite the expression of $C\left(k^{2}\right)$ as

$$
C\left(k^{2}\right) \equiv C_{3}\left(k^{2}\right)^{3}+C_{2}\left(k^{2}\right)^{2}+C_{1}\left(k^{2}\right)+C_{0},
$$

where $C_{3}=d_{1} d_{2} d_{3}>0$ for positive diffusion coefficients and $C_{2}>0$ as $C_{2}=$ $a_{11} N_{1}^{*} d_{2} d_{3}+a_{22} N_{2}^{*} d_{1} d_{3}+a_{33} N_{3}^{*} d_{1} d_{2}$. The consideration of $k=0$ reduces the characteristic Eq. (13) to the corresponding characteristic Eq. (7) of the associated 
non-spatial system and the local stability of $E_{*}$ for the non-spatial system is simply guaranteed by the inequalities presented in (8). As $A(0)=A=a_{11} N_{1}^{*}+a_{22} N_{2}^{*}+$ $a_{33} N_{3}^{*}>0$ holds true for any feasible coexistence steady state $E_{*}$, the conditions for temporal local stability of $E_{*}$ reduces to the inequalities $C(0)=C>0$ and $A(0) B(0)-C(0)=A B-C>0$. Hence, we obtain the condition $C_{0}>0$ as a necessary requirement for Turing instability where $C_{0}=C(0)=C=\left(a_{11} a_{22} a_{33}+\right.$ $\left.a_{12} a_{23} a_{31}+a_{13} a_{21} a_{32}-a_{11} a_{23} a_{32}-a_{12} a_{21} a_{33}-a_{13} a_{22} a_{31}\right) N_{1}^{*} N_{2}^{*} N_{3}^{*}$. Calculating the first and second order derivatives of $C\left(k^{2}\right)$, we obtain $\frac{d C\left(k^{2}\right)}{d\left(k^{2}\right)}=0$ and $\frac{d^{2} C\left(k^{2}\right)}{d\left(k^{2}\right)^{2}}>0$ at $k=k_{T}$. Therefore, the minimum for $C\left(k^{2}\right)$ occurs at $k=k_{T}$ where

$$
k_{T}^{2}=\frac{-C_{2}+\sqrt{C_{2}^{2}-3 C_{1} C_{3}}}{3 C_{3}} .
$$

Looking at Eq. (21), we can easily deduce that $k_{T}^{2}$ is a positive real number if $C_{1}<0$ or $C_{2}<0$ and $C_{2}^{2}>3 C_{1} C_{3}$. As we have already seen that $C_{2}>0$ holds true for our considered system, the condition responsible for the positivity of $k_{T}^{2}$ simply reduces to $C_{1}<0$. Therefore, we obtain the Turing bifurcation boundary as

$$
2 C_{2}^{3}-9 C_{1} C_{2} C_{3}-2\left(C_{2}^{2}-3 C_{1} C_{3}\right)^{\frac{3}{2}}+27 C_{0} C_{3}^{2}=0
$$

Note that Eq. (22) is free from the wavenumber $k$. We can consider it as an equation for the diffusion coefficient $d_{2}$ and accordingly, by solving it one can obtain the threshold value $d_{2}^{T}$ for the Turing instability whenever feasible positive solutions are available. However, the above equation is implicit and it is difficult to find $d_{2}^{T}$ analytically. Therefore, we compute $d_{2}^{T}$ numerically from Eq. (22) whenever all other parameter values are known.

Now, we present various numerical simulations which corroborate our theoretical results obtained in this subsection and illustrate other interesting spatiotemporal dynamics possessed by the spatial system (1-3). We would like to emphasize here that the conditions for Turing instability derived in this section are fairly general and accordingly, these conditions can be satisfied by several parameter sets satisfying both the cyclic and non-cyclic competitions. However, we are interested only in the dynamics of the system (1-3) with cyclic competition in this study. Therefore, we chose a parameter set in such a way that the inter-specific competitions follow a cyclic ordering. To be specific, here we are interested in parameter values which satisfy the conditions $a_{12}>a_{21}, a_{23}>a_{32}$ and $a_{31}>a_{13}$. For this purpose, we considered the following set of parameter values:

$$
\begin{aligned}
& r_{1}=2, r_{2}=2, r_{3}=2, a_{11}=0.8, a_{22}=1.2, a_{33}=0.8, a_{12}=1.2, a_{21}=0.7 \\
& a_{23}=1.2, a_{31}=1.2, a_{32}=0.7, d_{1}=0.1, d_{3}=0.2
\end{aligned}
$$

and varied the values of the parameters $a_{13}$ and $d_{2}$. For the chosen parameter values, Fig. 3 represents four different domains such as stable, Turing, Hopf-Turing and Hopf 
Fig. 3 Plots of temporal-Hopf and Turing bifurcation curves in $\left(a_{13}, d_{2}\right)$-parameter space for the reaction-diffusion system (1-3). The other parameter values are given in (23). The red vertical line and the blue dashed curve represent the temporal-Hopf threshold $\left(a_{13}^{*}=0.8\right)$ and the Turing bifurcation curve, respectively (Color Figure Online)

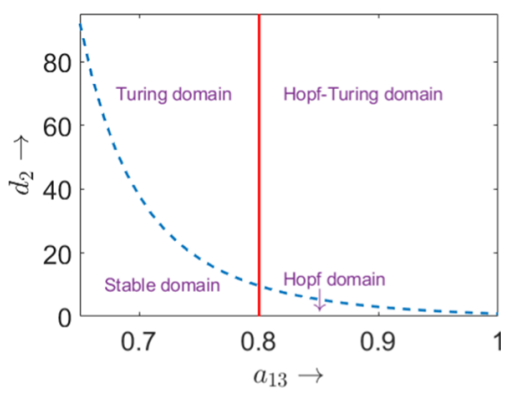

domains in the $\left(a_{13}, d_{2}\right)$-parameter space separated by the temporal-Hopf and Turing bifurcation curves.

For numerical simulations of the spatial system (1-3), we discretized the system using forward Euler scheme for the reaction part and central difference scheme for the diffusion part. The grid sizes (both the temporal and spatial) have been chosen in such a manner so that we can avoid the numerical artifacts. For all the numerical results presented in this subsection, we chose the computational domain as $[-200,200]$. Furthermore, the numerical simulations have been supplemented by periodic boundary conditions and the following pulse-type initial conditions:

$$
N_{j}(x, 0)=\left\{\begin{array}{l}
N_{j}^{*}+\eta_{j}, \quad|x|<10 \\
N_{j}^{*}, \quad 10 \leq|x| \leq 200
\end{array}\right.
$$

where $\left|\eta_{j}\right| \ll 1$ and $j=1,2,3$.

At this point, we exhibit various stationary and dynamic patterns which efficiently capture the rich spatiotemporal dynamics possessed by the spatial system (1-3). First, Fig. 4a represents the resulting population distribution of the first species $N_{1}$ for a representative point from the Turing domain presented in Fig. 3. We can observe that the pattern is stationary in time and heterogeneous in space, and this type of stationary population patches become apparent for reasonable rate of competition pressure exerted on the first species by the third with moderate dispersal rate of the second species. Other two competing species also sustain within similar stationary patches. Similarly, Fig. 4b represents the stationary spatially heterogeneous pattern of the population $N_{1}$ for a representative point lying in the Hopf-Turing domain. Note that the choice of different parameter values from both the Turing and HopfTuring domains will eventually lead to the stationary spatially heterogeneous pattern. However, we have observed that the patch size with high density increases for both the first and second species, and it decreases for the third one with increasing value of $d_{2}$. The reason behind this phenomenon can be attributed to the fact that successive two patches merge together and in turn, it leads to a decrease in the number of patches by increasing the width.

Figure $4 c-f$ demonstrates different types of dynamic patterns observed for the parameter values chosen from the Hopf domain. We do not include here the corresponding phase portraits of the spatially averaged densities of all the three competitive species (which are helpful for classification of the dynamic patterns) to make our main 


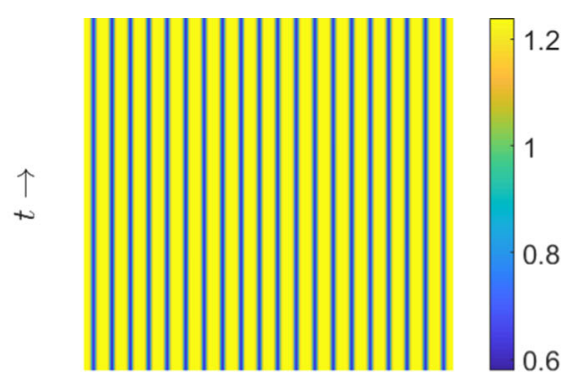

$x \rightarrow$

(a)

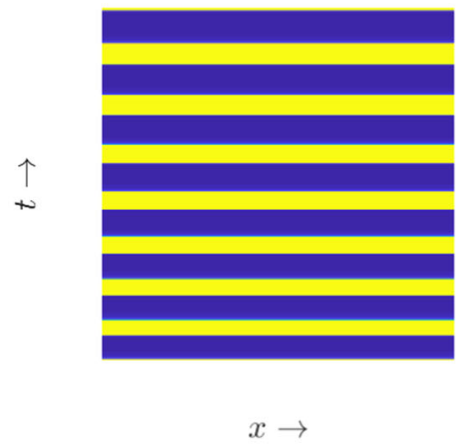

(c)

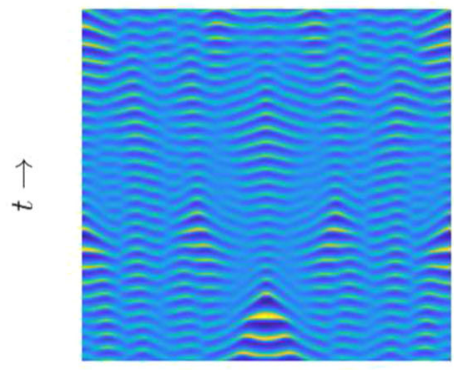

$x \rightarrow$

(e)

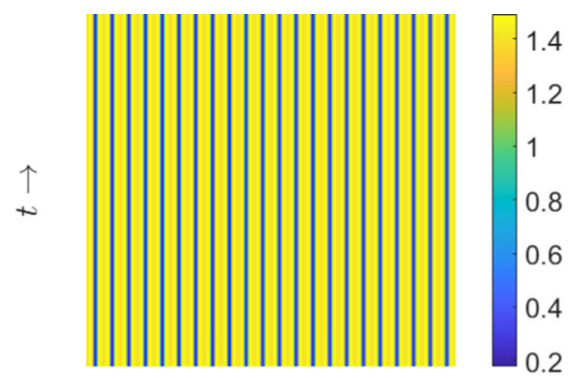

$x \rightarrow$

(b)

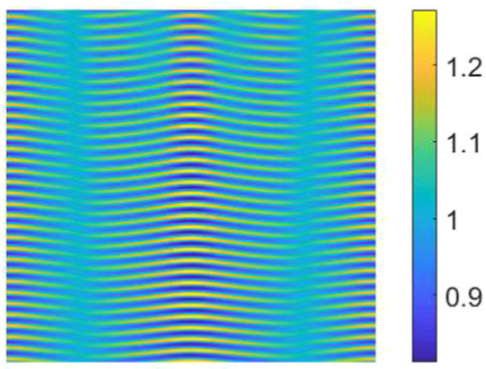

$x \rightarrow$

(d)

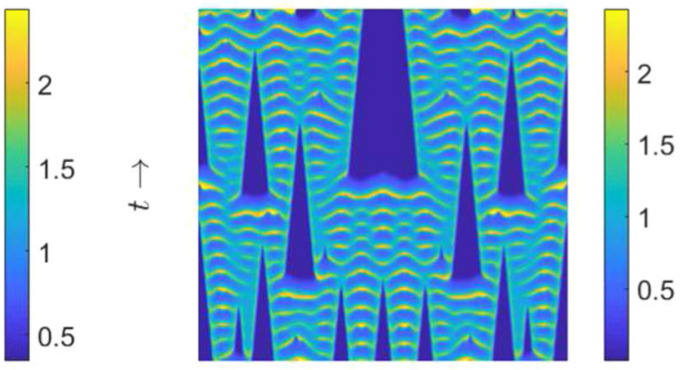

$x \rightarrow$

(f)

Fig. 4 Plots of various stationary and dynamic patterns exhibited by the first species $N_{1}$ for different values of the parameters $a_{13}$ and $d_{2}$. The patterns are obtained by simulating the spatial system (1-3) for a $a_{13}=0.75, d_{2}=25 ; \mathbf{b} a_{13}=0.85, d_{2}=10 ; \mathbf{c} a_{13}=0.8002, d_{2}=1 ; \mathbf{d} a_{13}=0.805, d_{2}=1$; e $a_{13}=0.85, d_{2}=1$; and $\mathbf{f} a_{13}=0.9, d_{2}=1$. The other parameter values are mentioned in (23). All the patterns are presented for a certain time period after neglecting the initial transients (Color Figure Online) 


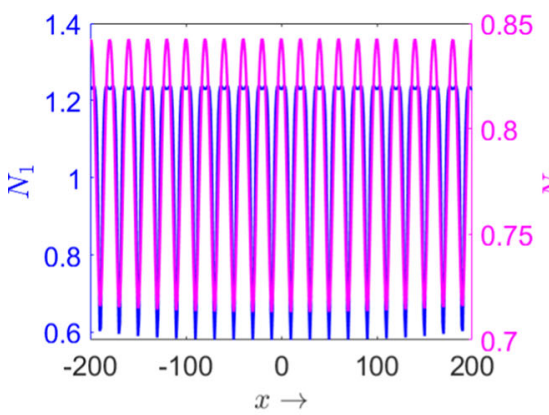

(a)

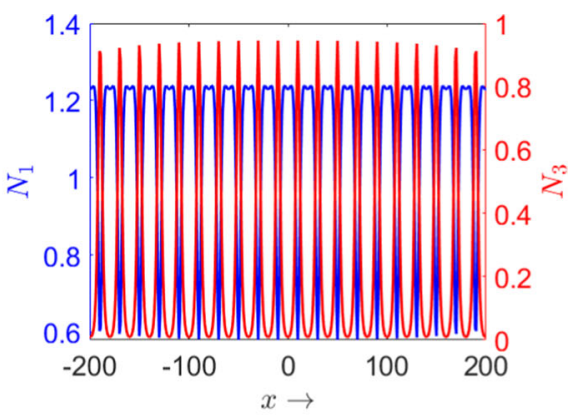

(b)

Fig. 5 Plots of spatial distribution of the densities of all the three competitive species. Here, left panel a illustrates the spatial distributions for first and second species, and right panel $\mathbf{b}$ illustrates the same for first and third species. The parameter values are the same as that for Fig. 4a. The plots are presented for a particular time instant after the population distributions settle in stationary regime (Color Figure Online)

text concise; however, we include them as Fig. 17 in Appendix A for the sake of clarity. In this regard, we fixed the value of $d_{2}$ to unity and varied the value of $a_{13}$. For gradual increments in the value of $a_{13}$ from the temporal-Hopf threshold, we obtained homogeneous in space and periodic in time (Fig. 4c), quasi-periodic (Fig. 4d), and chaotic (Fig. 4e and f) patterns. From Fig. 4c, we can easily observe that the period is not regular as it is expanding gradually with the advancement of time. This type of irregular periodic behavior in temporal scale has been demonstrated in May and Leonard (1975). For this pattern, the phase portrait describes a heteroclinic orbit joining the three single-species spatially homogeneous steady states (see Fig. 17a). Thus, the consideration of spatial mobility of all the three species transforms a Hopf-bifurcating stable limit cycle for the corresponding non-spatial model (see Fig. 1b) to a heteroclinic orbit. From ecological perspective, this indicates that moderate dispersal rates of all the three competing species destroy the stable oscillatory coexistence and lead to the extinction of two competing species. We call the pattern presented in Fig. $4 \mathrm{~d}$ a quasi-periodic pattern as the corresponding phase portrait is restricted to an annular region in the asymptotic sense (see Fig. 17b). In this case, the population distributions oscillate over both the space and time. Further, the phase portraits demonstrated in Fig. 17c and d effectively confirm the chaotic nature of the patterns presented in Fig. $4 \mathrm{e}$ and f, respectively. Also, we verified the chaotic nature by means of sensitivity to initial conditions, power spectrum and calculation of dominant Lyapunov exponent (Manna and Banerjee 2018; Mukherjee et al. 2018; Pascual 1993; Wolf et al. 1985), but restricted ourselves from including them in this study for the sake of brevity. The basic difference in these two chaotic patterns is that an interesting triangle-shaped structure emerges in Fig. 4f. Both the quasi-periodic and chaotic patterns suggest that the introduction of moderate mobility of all the three competing species restores the system which is doomed to collapse without any species mobility (see Fig. 1c and d) through the formation of dynamic population patches. Interestingly, these patterns are spatially symmetric with respect to the middle point of one-dimensional space. 


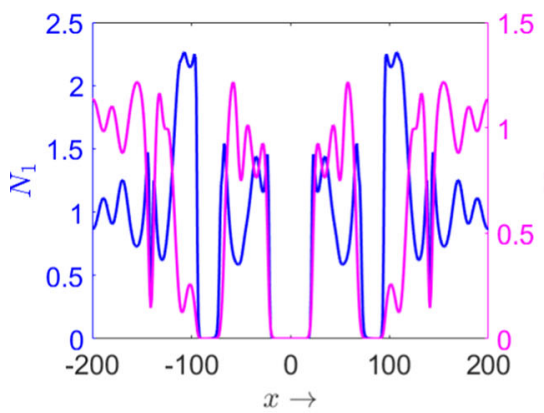

(a)

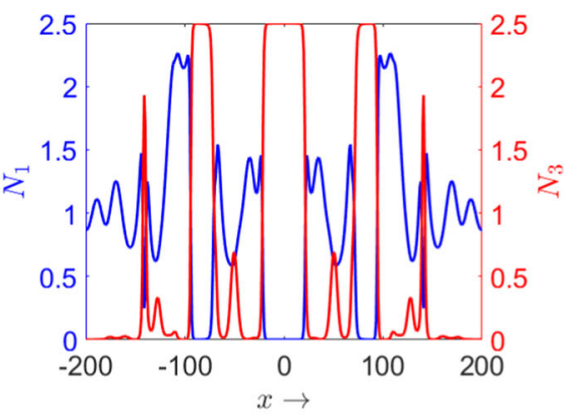

(b)

Fig. 6 Plots of spatial distribution of the densities of all the three competitive species. Here, left panel a illustrates the spatial distributions for first and second species, and right panel $\mathbf{b}$ illustrates the same for first and third species. The parameter values are the same as that for Fig. 4f. The plots are presented for a particular time instant after the population distributions settle in chaotic regime (Color Figure Online)

Figure 4 shows different types of patterns for the first species $N_{1}$ only as we have observed that the corresponding patterns for the remaining two competitive species (that is, $N_{2}$ and $N_{3}$ ) are qualitatively similar and accordingly, we restrict ourselves from displaying them in this study. However, the density correlations for all the three species differ and these differences are encapsulated in Figs. 5 and 6. For stationary patterns, we have observed that the high density patches of the first species $N_{1}$ correspond to the same as that of the second species $N_{2}$ and to the low density patches of the third species $N_{3}$. This phenomenon is illustrated in Fig. 5 with the same parameter values used for Fig. 4a. However, the density correlations change significantly for the corresponding dynamic spatially heterogeneous patterns. One such instance is shown in Fig. 6 with the same parameter values used for Fig. 4f.

The emergence of different types of stationary and dynamic patterns in different domains of the $\left(a_{13}, d_{2}\right)$-parameter space can also be characterized by the corresponding dispersion relations. Here, we present the typical dispersion relations corresponding to the four different domains by plotting the imaginary part and the maximum real part of the eigenvalues of the linearized system against a range of values of the wavenumber $k$ starting from zero. Accordingly, the five different representative dispersion relations are illustrated in Fig. 7. The five different parameter sets used to draw these dispersion relations give rise to a pair of complex conjugate eigenvalues for $k=0$ and then they become real for higher positive values of $k$ through a threshold $k_{*}$ which is denoted by a large black dot in each of these figures. First, we chose a representative parameter set from the stable domain and the resulting dispersion relation is presented in Fig. 7a. In this case, the largest real part of the eigenvalues stays negative over the entire admissible range of values of $k$, and accordingly, we could not find any spatiotemporal pattern. For this parameter set, we have $k_{*} \approx 0.269$ through which a pair of complex conjugate eigenvalues turn into real. Then, the dispersion relation for a representative parameter set from the Turing domain is presented in Fig. 7b. In this case, the largest real part of the eigenvalues becomes positive for the values of $k$ belonging to $\left(k_{-}, k_{+}\right)$with $k_{*}<k_{-}<k_{+}$, and accordingly, we found 


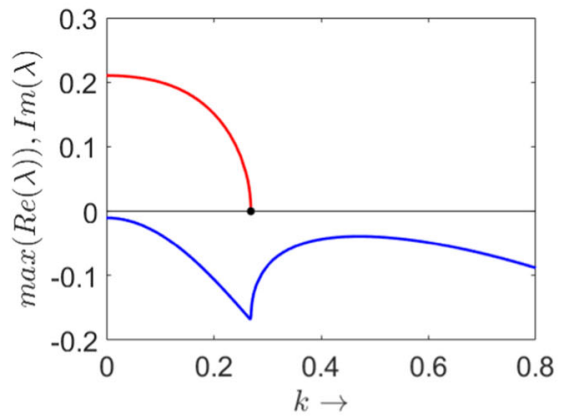

(a)

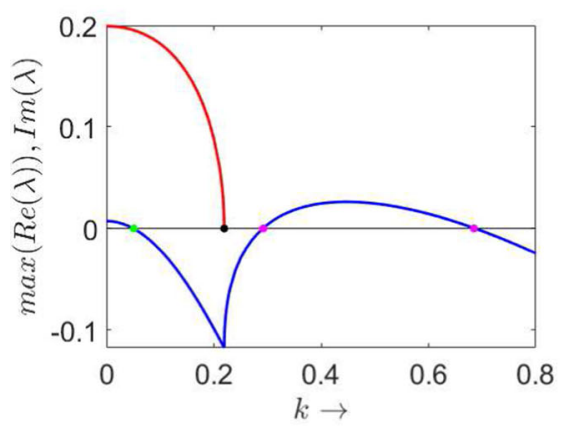

(c)

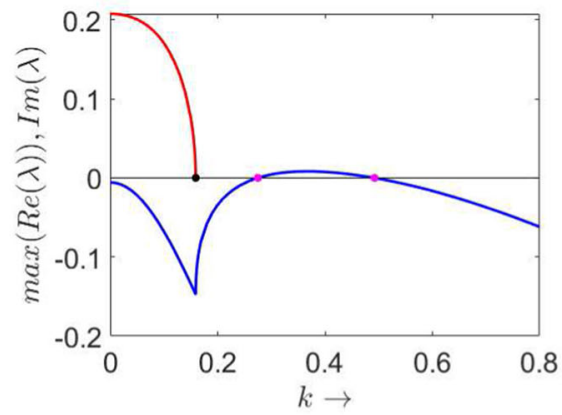

(b)

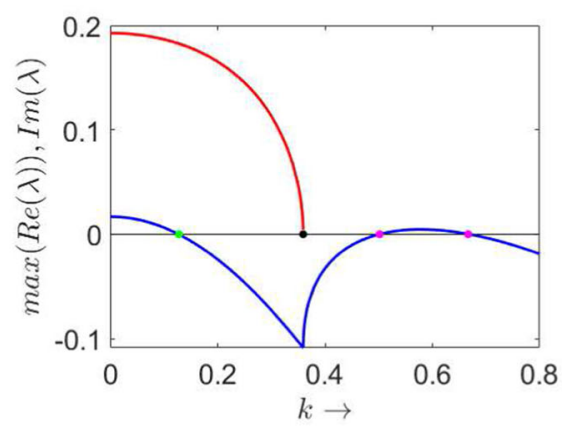

(d)

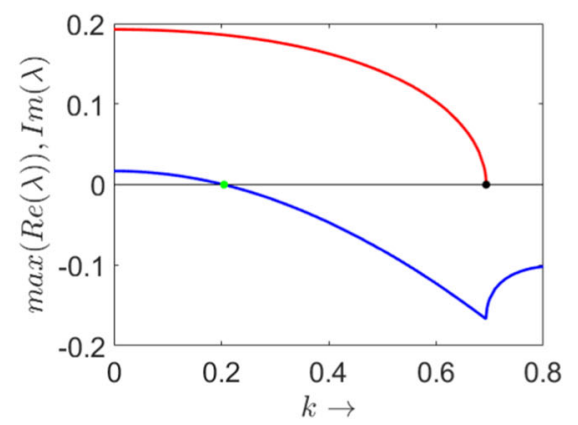

(e)

Fig. 7 Plots of dispersion relations of the spatial system (1-3) for different values of the parameters $a_{13}$ and $d_{2}$ : a $a_{13}=0.7, d_{2}=10 ; \mathbf{b} a_{13}=0.75, d_{2}=25 ; \mathbf{c} a_{13}=0.85, d_{2}=10 ; \mathbf{d} a_{13}=0.9, d_{2}=3.2$; e $a_{13}=0.9, d_{2}=1$. The other parameter values are given in (23). Imaginary parts and maximum of real parts of the eigenvalues against a range of values of the wavenumber $k$ are represented by red and blue colors, respectively (Color Figure Online)

a stationary Turing pattern (see Fig. 4a). For the considered parameter set, we have $k_{*} \approx 0.159, k_{-} \approx 0.275$ and $k_{+} \approx 0.493$. Both the $k_{-}$and $k_{+}$are denoted by large magenta dots in this figure.

Now, when the parameter $a_{13}$ is greater than the temporal-Hopf bifurcation threshold (that is, $a_{13}>a_{13}^{*}=0.8$ ), the largest real part of the eigenvalues becomes positive at $k=0$. Figure $7 \mathrm{c}$ and $\mathrm{d}$ demonstrates two possible representative dispersion relations 
for the parameter values chosen from the Hopf-Turing domain. In Fig. 7c, the largest real part of the eigenvalues remains positive in two disjoint sets of values of $k$, namely $\left[0, k_{1}\right)$ and $\left(k_{-}, k_{+}\right)$such that $k_{1}<k_{*}<k_{-}<k_{+}$. From this figure, we can observe that the largest real part in $\left(k_{-}, k_{+}\right)$is greater than the largest real part in $\left[0, k_{1}\right)$ and accordingly, we can expect the formation of stationary pattern which is corroborated by Fig. 4 b. For the chosen parameter set, we have $k_{1} \approx 0.051, k_{*} \approx 0.219, k_{-} \approx 0.292$ and $k_{+} \approx 0.685$. The quantity $k_{1}$ is indicated by a large green dot in this figure. Further in Fig. $7 \mathrm{~d}$, we have $k_{1} \approx 0.128, k_{*} \approx 0.36, k_{-} \approx 0.502$ and $k_{+} \approx 0.667$. Here, we can observe that the largest real part in $\left[0, k_{1}\right)$ is greater than the largest real part in $\left(k_{-}, k_{+}\right)$which is actually the opposite to that noticed in Fig. 7c. However, in this case, we obtained stationary spatially heterogeneous pattern for all the competing species and accordingly, did not include them in this manuscript for the sake of brevity. The dominating largest real part in $\left[0, k_{1}\right)$ produces only the dynamic transients which eventually settle into stationary spatially heterogeneous population distributions. The robustness of these stationary patterns has been verified with several different initial conditions. We would like to mention here that this dispersion relation (that is, Fig. 7d) can also correspond to some dynamic patterns in other ecological systems (Pal et al. 2020).

Finally, Fig. 7e demonstrates the dispersion relation for a representative parameter set from the Hopf domain. In this case, the largest real part is positive only in the interval $\left[0, k_{1}\right)$ and it stays negative for all $k>k_{1}$. This type of dispersion relation endorses the emergence of dynamic pattern and Fig. 4f serves as a concrete example. For this parameter set, we have $k_{1} \approx 0.205$ and $k_{*} \approx 0.694$. We would like to mention here that all other dynamic patterns presented in this subsection correspond to similar dispersion relations. However, the value of the maximal real part of the eigenvalues at $k=0$ plays a key role in determining the nature of the dynamic patterns. The population oscillations become more irregular for larger maximum real part of the eigenvalues at $k=0$.

An interesting phenomenon has been observed in the Hopf domain when the parameter values were taken very close to the Turing bifurcation curve. In this case, we obtained different types of dynamic patterns depending on the amplitudes of the pulsetype initial conditions. The simulation results are encapsulated in Fig. 8. The patterns presented here look very similar to each other; however, the differences in their nature can be understood from the corresponding phase portraits of the spatially averaged densities presented in Fig. 18 (see Appendix A). From these phase portraits, we classify the patterns presented in Fig. 8 as periodic, quasi-periodic and chaotic patterns, respectively. The emergence of different spatiotemporal population distributions for a fixed set of parameter values implies that the spatial system (1-3) admits multiple stable states. These patterns also demonstrate another interesting feature that the spatiotemporal oscillations are restricted in certain regions of the spatial domain, whereas the population distributions stay stationary over the remaining regions. However, the consideration of parameter values lying in the Hopf domain and sufficiently away from the Turing bifurcation curve leads to spatiotemporal oscillations over the whole domain for all the populations (see Fig. 4c-f). Note that the chosen set of parameter values gives rise to a qualitatively similar dispersion relation as presented in Fig. 7e. Further, taking different widths of the pulse-type initial conditions with fixed amplitude we 


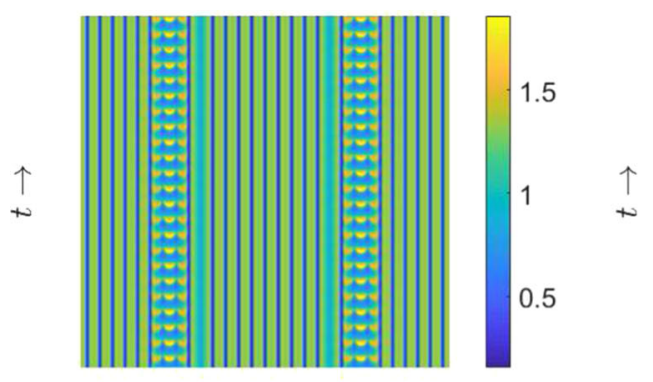

$x \rightarrow$

(a)

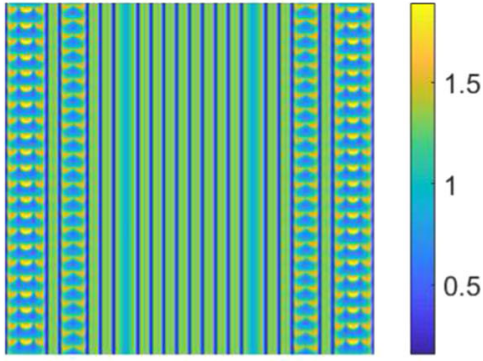

$x \rightarrow$

(b)

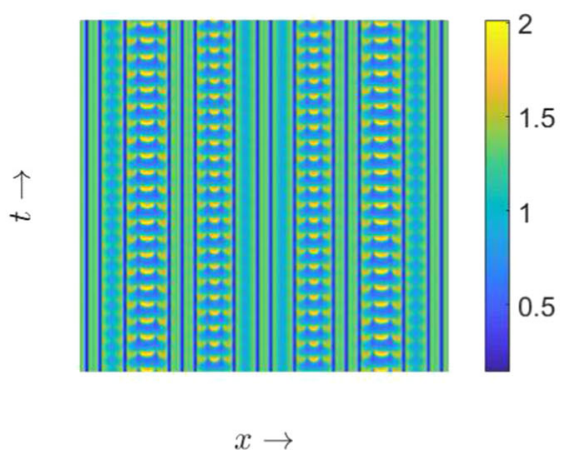

(c)

Fig. 8 Plots of different dynamic patterns such as a periodic, $\mathbf{b}$ quasi-periodic and $\mathbf{c}$ chaotic patterns exhibited by the first species $N_{1}$ for different amplitudes of the pulse-type initial conditions. The patterns are obtained by simulating the spatial system (1-3) for $a_{13}=0.9$ and $d_{2}=2.6$ with other parameter values as mentioned in (23). The patterns are presented for a certain time period after neglecting the initial transients (Color Figure Online)

obtained different dynamic patterns in this case as described above and accordingly, we did not include the simulation results in this study for the sake of brevity. Overall, these results signify that the spatiotemporal dynamics of the system (1-3) are highly sensitive to initial population distributions when the parameter values are taken from the Hopf domain and sufficiently close to the Turing bifurcation curve.

\subsection{Non-existence of Turing Patterns: A Special Case}

In this subsection, we aim to prove the non-existence of Turing patterns for the reaction-diffusion system (1-3) theoretically with specific form of kinetic parameters as considered in May and Leonard (1975). For this purpose, we assume the kinetic parameters as $r_{1}=r_{2}=r_{3}=1, a_{11}=a_{22}=a_{33}=1, a_{12}=a_{23}=a_{31}=\alpha$ and $a_{13}=a_{21}=a_{32}=\beta$ with $\alpha>0$ and $\beta>0$. With these types of kinetic parameters, the reaction-diffusion system (1-3) becomes 


$$
\begin{aligned}
& \frac{\partial N_{1}}{\partial t}=d_{1} \frac{\partial^{2} N_{1}}{\partial x^{2}}+\left(1-N_{1}-\alpha N_{2}-\beta N_{3}\right) N_{1}, \\
& \frac{\partial N_{2}}{\partial t}=d_{2} \frac{\partial^{2} N_{2}}{\partial x^{2}}+\left(1-\beta N_{1}-N_{2}-\alpha N_{3}\right) N_{2}, \\
& \frac{\partial N_{3}}{\partial t}=d_{3} \frac{\partial^{2} N_{3}}{\partial x^{2}}+\left(1-\alpha N_{1}-\beta N_{2}-N_{3}\right) N_{3},
\end{aligned}
$$

subjected to non-negative initial conditions and periodic boundary conditions. The spatiotemporal dynamics of this specific competition model (25-27) have been investigated elaborately in Adamson and Morozov (2012), Petrovskii et al. (2001). However, these studies did not discuss the stationary Turing patterns. Therefore, it would be really interesting to investigate the possible emergence of Turing patterns for this specific model. In this case, the spatially homogeneous coexistence steady state is given by

$$
E_{*} \equiv\left(\frac{1}{1+\alpha+\beta}, \frac{1}{1+\alpha+\beta}, \frac{1}{1+\alpha+\beta}\right)
$$

As local stability of $E_{*}$ for the non-spatial system is a necessary requirement for the emergence of Turing instability in the spatial system, we briefly reiterate the condition regarding the stability of $E_{*}$ in non-spatial setting. One can go through the reference (May and Leonard 1975) for the detailed investigation of the dynamics of the corresponding non-spatial system. The coexistence steady state $E_{*}$ is locally stable if the inequality $\alpha+\beta<2$ is satisfied (May and Leonard 1975). Thus, by employing the AM-GM inequality for positive real numbers we obtain

$$
\sqrt{\alpha \beta} \leq \frac{\alpha+\beta}{2}<1 \Rightarrow \alpha \beta<1
$$

Now, the characteristic equation for the spatial model (25-27) is given by

$$
\lambda^{3}+A\left(k^{2}\right) \lambda^{2}+B\left(k^{2}\right) \lambda+C\left(k^{2}\right)=0,
$$

where

$$
\begin{aligned}
A\left(k^{2}\right)= & \left(d_{1}+d_{2}+d_{3}\right) k^{2}+\frac{3}{(1+\alpha+\beta)}, \\
B\left(k^{2}\right)= & \left(d_{1} d_{2}+d_{2} d_{3}+d_{3} d_{1}\right) k^{4}+\frac{2\left(d_{1}+d_{2}+d_{3}\right)}{1+\alpha+\beta} k^{2}+\frac{(1-\alpha \beta)}{(1+\alpha+\beta)^{2}}, \\
C\left(k^{2}\right)= & d_{1} d_{2} d_{3} k^{6}+\frac{\left(d_{1} d_{2}+d_{2} d_{3}+d_{3} d_{1}\right)}{(1+\alpha+\beta)} k^{4}+\frac{(1-\alpha \beta)\left(d_{1}+d_{2}+d_{3}\right)}{(1+\alpha+\beta)^{2}} k^{2} \\
& +\frac{\left(1+\alpha^{3}+\beta^{3}-3 \alpha \beta\right)}{(1+\alpha+\beta)^{3}} .
\end{aligned}
$$


Fig. 9 Plot of chaotic pattern exhibited by the first species $N_{1}$ for the specific spatial system (25-27). Here, the used parameter values are $\alpha=0.8$, $\beta=1.9, d_{1}=0.1, d_{2}=1$ and $d_{3}=0.2$. The pattern is presented for a certain time period after neglecting the initial transients (Color Figure Online)

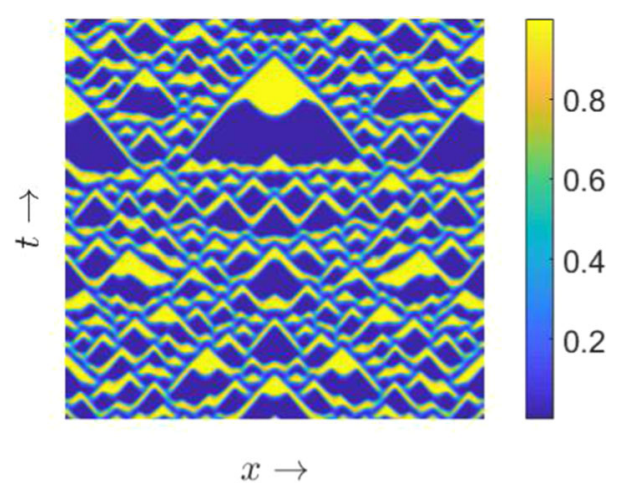

From the analysis performed in the preceding subsection, we can easily obtain in this case that the condition for positivity of the quantity $k_{T}^{2}$ reduces to

$$
C_{1}=\frac{(1-\alpha \beta)\left(d_{1}+d_{2}+d_{3}\right)}{(1+\alpha+\beta)^{2}}<0 \Rightarrow \alpha \beta>1 \text {. }
$$

But the inequality (30) contradicts the inequality obtained in (28). Therefore, we do not have any positive $k_{T}^{2}$ in order to induce Turing instability and then we should not expect any stationary Turing patterns for this specific competition model (25-27). Also, we have performed several numerical simulations which support our theoretical claim and restrict ourselves from incorporating them in this manuscript for the sake of brevity.

We have already proved in this subsection that the specific spatial system (25-27) does not admit any Turing pattern; however, it can possess a rich variety of dynamic non-Turing patterns. Spatiotemporal dynamics of this model have been investigated quite thoroughly in the studies (Adamson and Morozov 2012; Petrovskii et al. 2001). Both the references (Adamson and Morozov 2012; Petrovskii et al. 2001) demonstrated the existence of traveling waves over one-dimensional spatial domain, and the existence of spirals, spiraling patches, chaotic patches and co-invasion over twodimensional spatial domain. However, there exist clear distinctions in the choice of initial and boundary conditions used for the numerical simulations of our study with the references (Adamson and Morozov 2012; Petrovskii et al. 2001). Invasion-type initial conditions were used in Adamson and Morozov (2012), Petrovskii et al. (2001), while we relied on the pulse-type initial conditions mentioned in (24). Adamson and Morozov (2012) considered the invasion-type initial conditions where the first two species occupy two non-overlapping patches and the third species is present in the remaining domain at their respective environmental carrying capacities. On the other hand, Petrovskii et al. (2001) chose non-zero population densities of the three species at some regions in the middle of the domain with zero population densities elsewhere. Also, we adopted periodic boundary conditions in contrast to no-flux boundary conditions used in Adamson and Morozov (2012), Petrovskii et al. (2001). Performing several numerical simulations of the system (25-27), we observed a similar transition of dynamic patterns from homogeneous in space and periodic in time pattern to chaotic 
pattern through quasi-periodic one for increasing $\beta$ as illustrated in Fig. 4. In this case, Fig. 9 represents the chaotic population distribution of the first species. Note that this pattern is qualitatively similar to the chaotic patterns presented in Fig. 4; however, the size and scale of the patches are quite different. Further, for a sufficiently large value of $d_{2}$ the system can eventually collapse where two competitive species go to extinction and the remaining species persists at its environmental carrying capacity.

\section{Nonlocal Reaction-Diffusion System}

The spatial system (1-3) presented in the preceding section has assumed that an individual of a particular species located at a specific spatial point interacts with other individuals of that species present at that point only and hence, considered local intra-specific interactions. However, individuals from a species can interact with each other inside a suitable neighborhood due to the spatial mobility of that species. This phenomenon gives rise to the nonlocal intra-specific interactions. In this section, we incorporate nonlocal interaction terms taking into account the intra-specific competition for the resources in the reaction-diffusion system (1-3) and accordingly, extend the model. Note that the mechanisms such as fights, cannibalism, space sharing etc. can also potentially lead to nonlocal intra-specific competition apart from the usual resource consumption. The nonlocal cyclic competition model is given by

$$
\begin{aligned}
& \frac{\partial N_{1}}{\partial t}=d_{1} \frac{\partial^{2} N_{1}}{\partial x^{2}}+\left(r_{1}-a_{11} J_{1}\left(N_{1}\right)-a_{12} N_{2}-a_{13} N_{3}\right) N_{1}, \\
& \frac{\partial N_{2}}{\partial t}=d_{2} \frac{\partial^{2} N_{2}}{\partial x^{2}}+\left(r_{2}-a_{21} N_{1}-a_{22} J_{2}\left(N_{2}\right)-a_{23} N_{3}\right) N_{2}, \\
& \frac{\partial N_{3}}{\partial t}=d_{3} \frac{\partial^{2} N_{3}}{\partial x^{2}}+\left(r_{3}-a_{31} N_{1}-a_{32} N_{2}-a_{33} J_{3}\left(N_{3}\right)\right) N_{3},
\end{aligned}
$$

subjected to non-negative initial conditions and periodic boundary conditions. Here, the nonlocal intra-specific competitions have been taken care of by $J_{S}\left(N_{S}\right)(s=$ $1,2,3)$, where

$$
J_{s}\left(N_{s}\right)=\int_{-\infty}^{\infty} \phi_{s}(x-y) N_{s}(y, t) d y
$$

with

$$
\phi_{s}(z)=\left\{\begin{array}{ll}
\frac{1}{2 \delta_{s}}, & |z| \leq \delta_{s} \\
0, & \text { elsewhere }
\end{array} .\right.
$$

For the sake of simplicity, we have taken the kernel functions $\phi_{s}(s=1,2,3)$ as the top-hat kernels. This type of kernel function is non-negative and even which efficiently captures the unbiased movement of all the three competitive species. Also, the parameters $\delta_{s}(s=1,2,3)$ involved in the kernel functions $\phi_{s}$ signify the extent of nonlocality as they regulate the width of the kernels. For a kernel function $\phi_{s}$ as 


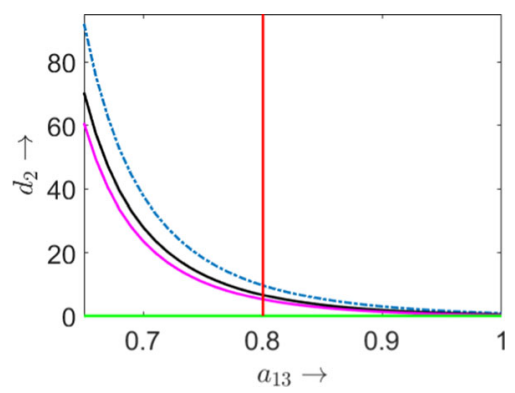

Fig. 10 Plots of temporal-Hopf and Turing bifurcation curves in $\left(a_{13}, d_{2}\right)$-parameter space for different values of the extent of nonlocality $\delta\left(\delta_{1}=\delta_{2}=\delta_{3}=\delta\right)$ for the nonlocal system (31-33). The red vertical line represents the temporal-Hopf threshold $\left(a_{13}^{*}=0.8\right)$. The Turing bifurcation curves for different values of $\delta$ are represented by blue $(\delta=0.001)$, black $(\delta=1)$, magenta $(\delta=1.2)$ and green $(\delta=2)$ colored curves, respectively. The other parameter values are given in (23) (Color Figure Online)

defined above, the nonlocal interaction is restricted within an one-dimensional spatial region of length $2 \delta_{s}$ where the efficacy of it remains constant. Further, we can easily deduce that $\int_{-\infty}^{\infty} \phi_{S}(z) d z=1$. Therefore, the nonlocal system (31-33) admits the same spatially homogeneous steady states as that of the reaction-diffusion system (1-3) and accordingly, we denote them by using the same notations. One can easily derive the conditions for Turing instability of the nonlocal system (31-33) following the procedure presented in Sect. 2.2. However, we restrict ourselves from incorporating the derivation of these conditions here for the sake of smooth readability. The detailed derivation of these conditions is provided in the Supplementary material.

Now, we investigate numerically the effect of the extent of nonlocality on the spatiotemporal dynamics possessed by the reaction-diffusion system (1-3). For the sake of simplicity, we consider the same extent of nonlocality for all the three competitive species (that is, $\delta_{1}=\delta_{2}=\delta_{3}=\delta$ ). First, we examine how the different values of $\delta$ act on the Turing bifurcation curve with the parameter values given in (23). Some of these scenarios are illustrated in Fig. 10 where we can observe the relative positions of the Turing bifurcation curves for different values of $\delta$. It is a well-known fact that the nonlocal system (31-33) becomes the local reaction-diffusion system (1-3) as $\delta \rightarrow 0+$. Thus, the Turing bifurcation curves for both the local and nonlocal systems become the same as $\delta \rightarrow 0+$. This phenomenon is illustrated in Fig. 10 by the blue broken curve which is exactly the same with the Turing bifurcation curve presented in Fig. 3. The other Turing bifurcation curves illustrated in Fig. 10 (see black and magenta curves) show that the increased value of $\delta$ extends both the Turing and Hopf-Turing domains by diminishing the other two domains (stable and Hopf domains). Further, a sufficiently large value of $\delta$ eventually leads to almost disappearance of both the stable and Hopf domains, and the whole $\left(a_{13}, d_{2}\right)$-parameter space is divided into the Turing and Hopf-Turing domains (see green curve in Fig. 10).

Now, we present some patterns accounting for the spatiotemporal dynamics of the nonlocal system (31-33). In order to numerically simulate the system (31-33), we used forward Euler method, trapezoidal rule and central difference scheme for the reaction parts, nonlocal interaction terms and diffusion parts, respectively. The 


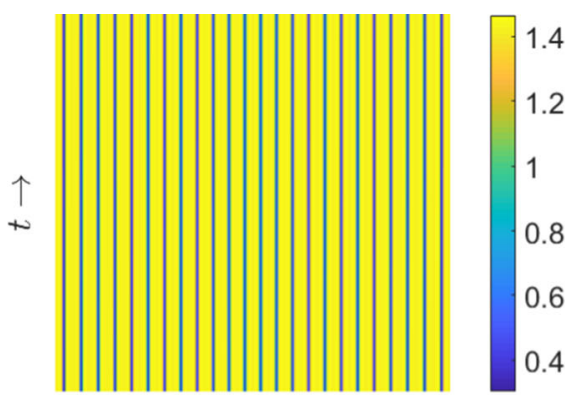

$x \rightarrow$

(a)

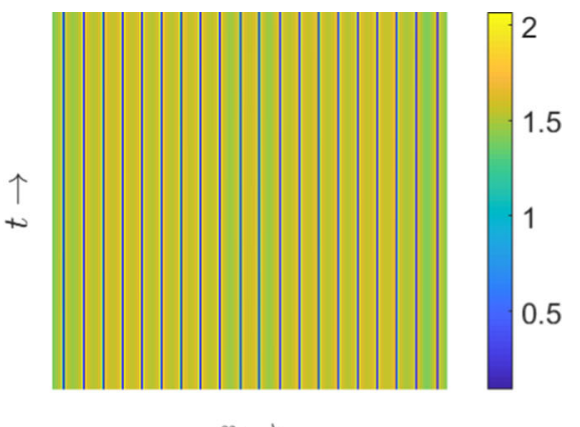

$x \rightarrow$

(b)

Fig. 11 Plots of stationary Turing patterns accounting for the effect of the extent of nonlocality $(\delta)$ for the first species $N_{1}$. The patterns are obtained by simulating the nonlocal system (31-33) for $\mathbf{a} \delta=1.0$ and $\mathbf{b}$ $\delta=1.5$. The other parameter values are mentioned in (23) with $a_{13}=0.75$ and $d_{2}=25$. The patterns are presented for a certain time period after neglecting the initial transients (Color Figure Online)

numerical simulations were carried out in an one-dimensional computational spatial domain $[-200,200]$ with the periodic boundary conditions and the pulse-type initial conditions as mentioned in (24). Further, both the spatial and temporal grid sizes have been chosen appropriately to avoid any sorts of numerical artifacts.

First, we investigate numerically the effect of the extent of nonlocality on the stationary Turing pattern presented in Fig. 4a and the resulting patterns are illustrated in Fig. 11. The figures show that incorporation of nonlocal intra-specific interaction does not possess any drastic effect on the dynamics of the system and retains the stationary nature of the pattern which can be expected from the positioning of the Turing bifurcation curves presented in Fig. 10. However, the variation in population density becomes larger with the increment in the value of $\delta$ which is evident from the respective color bars. We would like to mention at this stage that one can observe similar impact of $\delta$ on other stationary Turing and non-Turing patterns possessed by the local reaction-diffusion system (1-3).

Further, we investigate numerically the effect of the extent of nonlocality on the chaotic pattern presented in Fig. $4 \mathrm{f}$ and the resulting patterns are illustrated in Fig. 12. Our simulation result presented in Fig. 12a shows that the nonlocal model possesses the triangle-shaped chaotic pattern similar to that of the local model; however, the spatial symmetry about the middle point is destroyed for moderate extent of nonlocality. This symmetry-breaking capability of the extent of nonlocality has been documented previously in Manna et al. (2020). Further increment in the value of $\delta$ demolishes the triangle-like structure, while retains the chaotic nature as shown in Fig. 12b. In this figure, one can observe that stationary structure emerges in middle region with chaotic structure toward the boundaries. Finally, sufficiently large value of $\delta$ transforms the chaotic pattern to a stationary one (see Fig. 12c). This type of transition from a dynamic pattern to a stationary one can be observed for other dynamic patterns possessed by the local model (1-3).

Another interesting phenomenon has been observed for the population distribution presented in Fig. 12c where the second competitive species goes to extinction due 


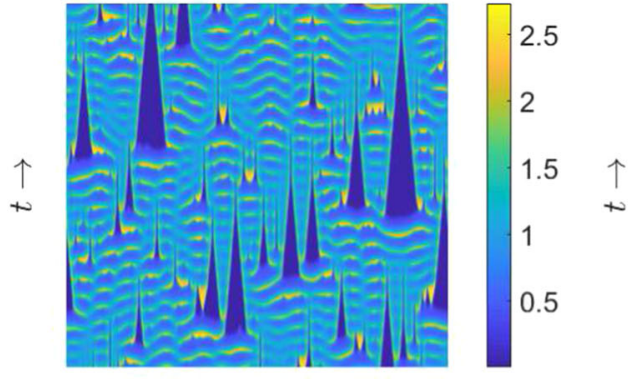

$x \rightarrow$

(a)

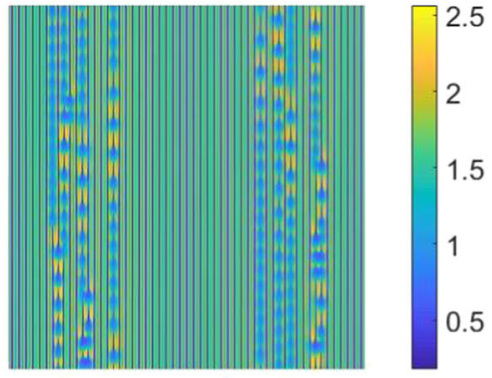

$x \rightarrow$

(b)

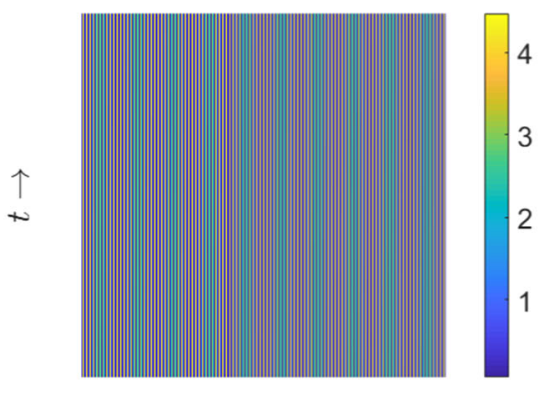

$x \rightarrow$

(c)

Fig. 12 Plots of different types of dynamic and stationary patterns accounting for the effect of the extent of nonlocality $(\delta)$ for the first species $N_{1}$. The patterns are obtained by simulating the nonlocal system (31-33) for $\mathbf{a} \delta=1.0, \mathbf{b} \delta=1.2$ and $\mathbf{c} \delta=1.5$. The other parameter values are mentioned in (23) with $a_{13}=0.9$ and $d_{2}=1$. The patterns are presented for a certain time period after neglecting the initial transients (Color Figure Online)

\section{Fig. 13 Plot of spatial}

distribution of the densities for the first and third species. The parameter values are the same as that for Fig. 12c. Note that the spatial distributions are presented over the spatial domain $[-50,50]$ for the clarity of visualization though the simulation was carried out over the domain $[-200,200]$. The plot is presented for a particular time instant after the population distributions settle in stationary regime (Color Figure Online)

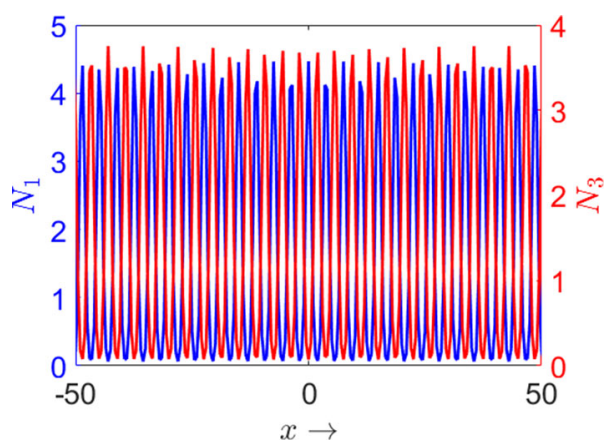




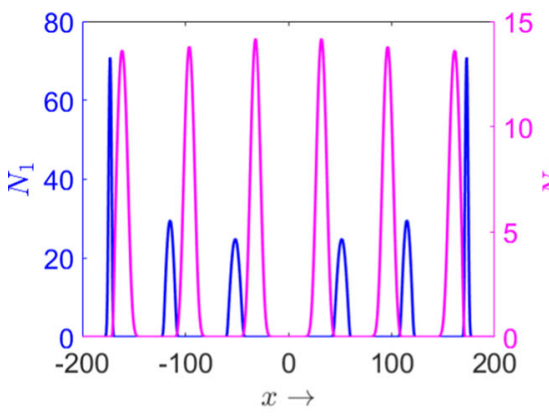

(a)

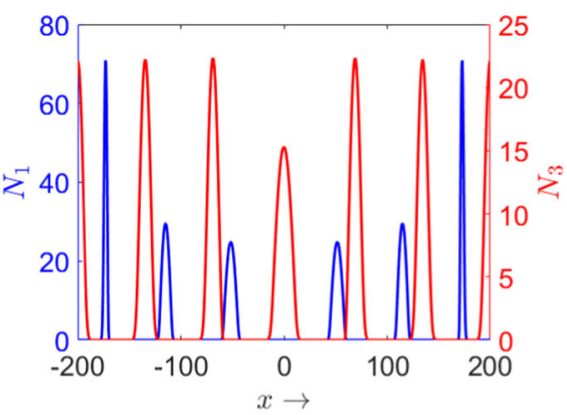

(b)

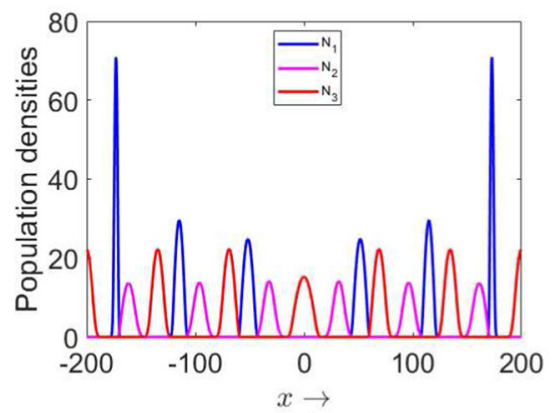

(c)

Fig. 14 Plots of spatial distribution of the densities of all the three competitive species. Here, the panel a illustrates the spatial distributions for first and second species, and the panel $\mathbf{b}$ illustrates the same for first and third species. The panel $\mathbf{c}$ illustrates the spatial distributions for all the three species. The plots are obtained by simulating the nonlocal system (31-33) for the parameter values mentioned in (23) with $a_{13}=0.9, d_{2}=1$ and $\delta=50$. The plots are presented for a particular time instant after the population distributions settle in stationary regime (Color Figure Online)

to huge competition pressure exerted by other two species. This phenomenon can be easily explained from the density correlation between the first and third species presented in Fig. 13. From this density correlation plot, we can notice that both the first and third species aggregate in large numbers over the entire habitat, and hence, exert an immense competition pressure on the remaining species so that the species goes to extinction. Further increments in the value of $\delta$ up to a certain extent retain this scenario while the number of patches for both the first and third species decreases gradually, aggregation in respective patches increases, and patches corresponding to a species move away from each other. After a certain threshold value of $\delta$, the second species can persist in stationary patches along with other two competitive species. One such numerical example is provided in Fig. 14. This phenomenon arises as the patches corresponding to the first and third species align in such a way that the second species can access some empty space for resources within their habitat. In this case, all the three species survive in completely separate patches. Therefore, the incorporation of nonlocal intra-specific interaction has a negative feedback on the persistence of the second species, while it has positive feedback on the persistence of the remaining 


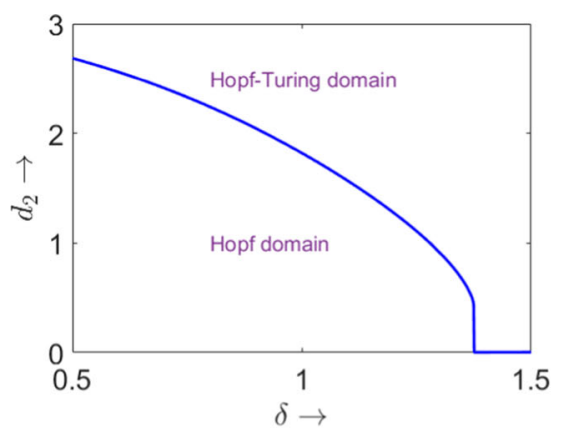

(a)

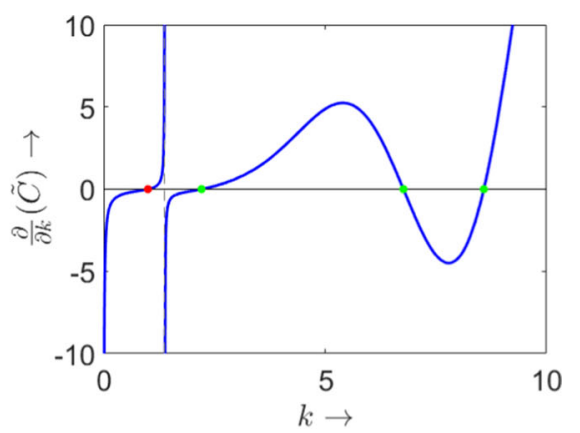

(b)

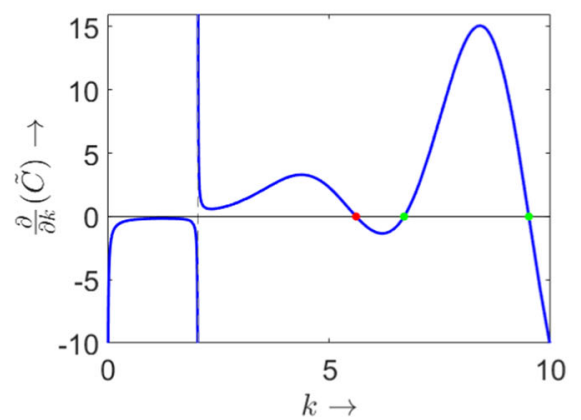

(c)

Fig. 15 Plot of the Turing bifurcation curve in $\left(\delta, d_{2}\right)$-parameter space for $a_{13}=0.9$ (panel a). Plots of the function $\frac{\partial \tilde{C}}{\partial k}$ for $\delta=1.2$ (panel b) and $\delta=1.5$ (panel $\mathbf{c}$ ). The other parameter values are mentioned in (23) (Color Figure Online)

two species for a certain range of values of $\delta$. Overall, the system collapses for an intermediate range of values of $\delta$ and the bio-diversity can be restored either in dynamic or stationary mode outside this range. It is worthy to mention here that this type of nonlocality-driven loss and regeneration of bio-diversity has been observed for all other stationary and dynamic patterns possessed by the local model (1-3).

The transition of the patterns from chaotic to stationary as presented in Fig. 12 can be explained efficiently from the plot of the corresponding Turing bifurcation curve in $\left(\delta, d_{2}\right)$-parameter space (see Fig. 15a). In this case, the Turing bifurcation curve divides the $\left(\delta, d_{2}\right)$-parameter space into the Hopf-Turing and Hopf domains. From this figure, we can observe that the parameter sets used for the dynamic patterns presented in Fig. 12a and $b$ lie in the Hopf domain and accordingly, one should expect the emergence of dynamic patterns. However, the parameter set used for the stationary pattern illustrated in Fig. 12c lies well inside the Hopf-Turing domain and this explains the emergence of stationary pattern. Interestingly, the Turing bifurcation curve is not smooth in this case and a point of non-differentiability arises at $\delta=1.376$. Thus, it would be reasonable to look for an explanation for the emergence of this point of non-differentiability. In order to explain this feature of the Turing bifurcation curve, we plot the function $\frac{\partial \tilde{C}}{\partial k}$ 
Fig. 16 Plot of stationary spatially heterogeneous pattern for the first species $N_{1}$. The pattern is obtained by simulating the nonlocal system (31-33) for the parameter values

$r_{1}=r_{2}=r_{3}=1$,

$a_{11}=a_{22}=a_{33}=1$,

$a_{12}=a_{23}=a_{31}=\alpha=0.8$,

$a_{13}=a_{21}=a_{32}=\beta=1$,

$d_{1}=0.1, d_{2}=0.5, d_{3}=0.2$

and $\delta=2$. The pattern is

presented for a certain time

period after neglecting the initial

transients (Color Figure Online)

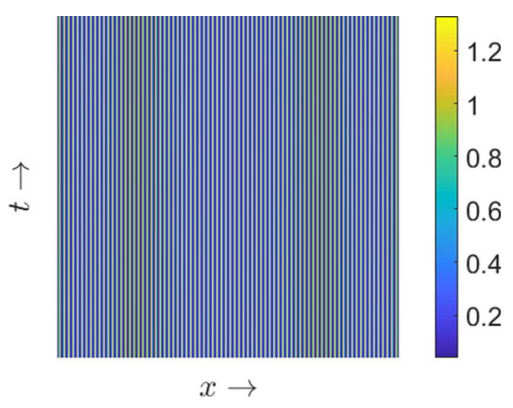

against the wavenumber $k$ for $\delta=1.2$ and 1.5 (see Fig. 15b and c), and look for the first positive root of it in terms of $k$ which describes the critical wavenumber $k_{T}$ responsible for the Turing instability. The critical wavenumbers $k_{T}$ are indicated by large red dots in the corresponding figures, whereas other positive roots are indicated by large green dots. Note that the function $\frac{\partial \tilde{C}}{\partial k}$ admits infinitely many positive roots; however, we have presented the corresponding figures with first few positive roots for a reasonable numerical illustration. In both of these figures, we can observe the existence of asymptotes for positive $k$-values (at $k=1.379$ for $\delta=1.2$ and at $k=2.04$ for $\delta=1.5$ ). For $\delta=1.2$, we have obtained $k_{T}=1.001$ which lies in the left-side of the asymptote. However, the critical wavenumber $k_{T}=5.611$ lies in the right-side of the asymptote when $\delta=1.5$. Therefore, the point of non-differentiability arises as the critical wavenumber $k_{T}$ crosses the asymptote from left to right at $\delta=1.376$.

We have already proved in the preceding section that the specific spatial system (25-27) does not admit any stationary Turing pattern. We could not be able to find any stationary non-Turing pattern through numerical simulations neither. Therefore, it would be interesting to examine numerically whether the incorporation of nonlocal intra-specific interactions can induce any stationary spatially heterogeneous population distribution. Through numerical investigations, we have observed that the incorporation of nonlocality in intra-specific interactions can effectively drive stationary spatially uniform population distribution to become stationary spatially heterogeneous one. However, moderate extent of nonlocality leads to the extinction of the second species and the remaining two species survive with stationary population patches (see Fig. 16). The reasoning of this type of bio-diversity loss has been provided in the previous paragraph. Further, the bio-diversity can be regained for sufficiently large value of $\delta$ as shown in Fig. 14. For the dynamic patterns (for example, the chaotic pattern presented in Fig. 9) possessed by the spatial system (25-27), we have observed a similar kind of transition from dynamic to stationary spatial population distributions depending on the extent of nonlocality as illustrated in Figs. 12 and 14. Accordingly, we restrict ourselves from incorporating these results in this study for the sake of brevity. 


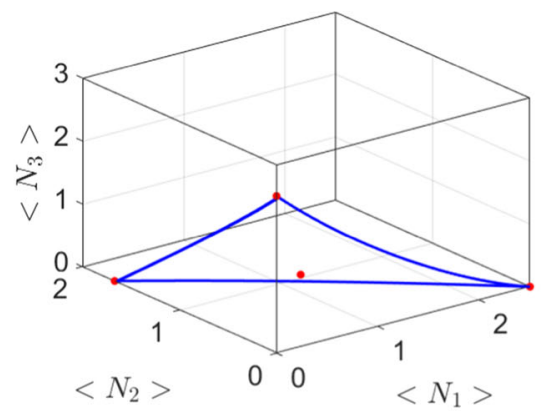

(a)

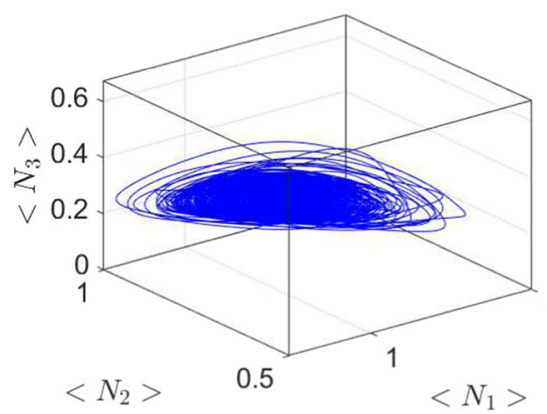

(c)

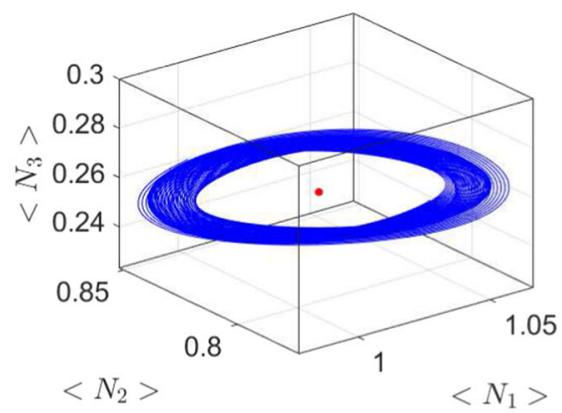

(b)

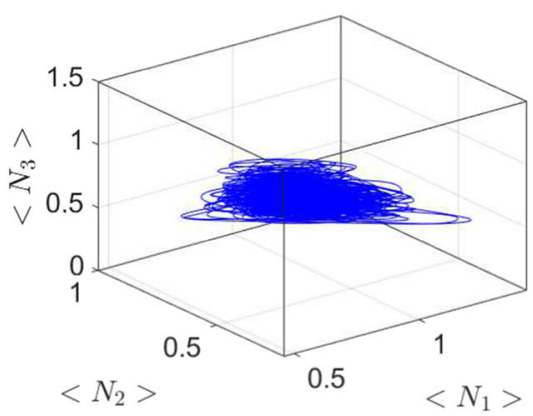

(d)

Fig. 17 Phase portraits of the spatially averaged densities of all the three competitive species corresponding to the dynamic patterns presented in Fig. 4 (that is, Figs. 4c-f) (Color Figure Online)

\section{Discussion and Conclusions}

In the existing literature, we find only a few studies on the spatiotemporal dynamics of competition models for ecological populations taking into account the formation of various stationary and non-stationary patchy patterns. However, there does not exist any study which demonstrates the spatiotemporal dynamics of a three-species competition model with both self-diffusion and nonlocal intra-specific competitions. In this paper, we have investigated the rich spatiotemporal dynamics of a LotkaVolterra type three-species cyclic competition model with self-diffusion and nonlocal intra-specific competitions.

Firstly, we have summarized a wide variety of dynamics possessed by the corresponding non-spatial system (4-6) in terms of both the analytical and numerical results. Numerical simulations with parameter values accounting for the specific cyclic ordering where $a_{12}>a_{21}, a_{23}>a_{32}$ and $a_{31}>a_{13}$ reveal that the stable steady state coexistence changes to the stable oscillatory coexistence for increased competition pressure exerted by the third species on the first species (that is, $a_{13}$ ). Further increment in the same competition strength leads to the system collapse through a global bifurcation such as homoclinic bifurcation and only the third species survives at its carrying capacity. From ecological point of view, larger values of $a_{13}$ make the third 


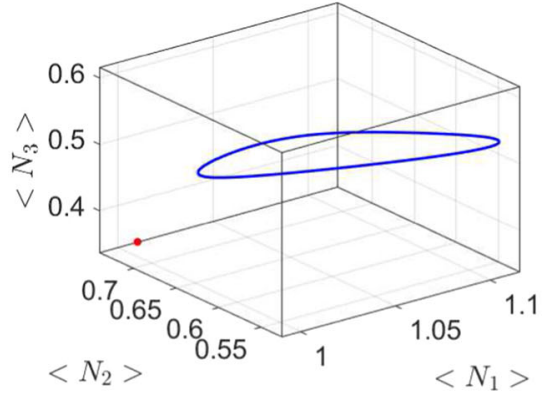

(a)

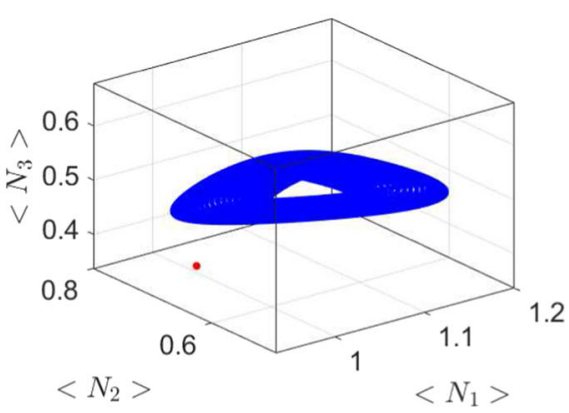

(b)

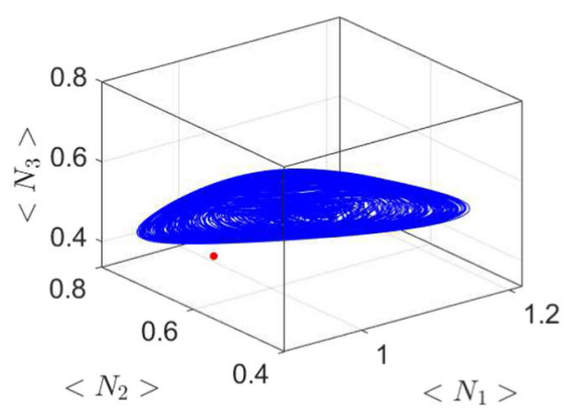

(c)

Fig. 18 Phase portraits of the spatially averaged densities of all the three competitive species corresponding to the dynamic patterns presented in Fig. 8 (Color Figure Online)

species a superior competitor and accordingly, the third species emerges as sole survivor by destroying the stable oscillatory coexistence for sufficiently large value of $a_{13}$.

Further, numerical simulations for the parameter values accounting for another cyclic ordering where $a_{12}<a_{21}, a_{23}<a_{32}$ and $a_{31}<a_{13}$ show that the stable oscillatory coexistence disappears through the heteroclinic bifurcation for a sufficiently large value of the parameter $\beta\left(\beta=a_{13}=a_{21}=a_{32}\right)$. The parameter $\beta$ represents the competition pressure exerted by one species to another following this cyclic ordering. Thus, the increased value of $\beta$ does not make a specific species a superior competitor over the others; however, it makes a species better competitor than another following this cyclic ordering. This is the reason why the survival of one species depends on the initial population densities. Extinction of one or more species due to competitive exclusion is a landmark characteristic for multi-species competition models. The extinction scenario is determined by the intra- and inter-specific competition strengths for the models with homogeneous population distribution.

Our findings regarding the formation of stationary patches in the presence of selfdiffusion of all the competing species supplement the studies conducted in Kishimoto (1982), Martínez (2003). For mathematical tractability we often consider the system consisting with less number of species, but in reality, we find multi-component species 
competing for the same resource and habitat. Therefore, our study reveals that the existence of stationary spatially heterogeneous population distributions is more likely to be observed in nature, and explains the formation of population patches with multiple competitive species when the strength of competitions is moderate, the resources are adequate and the dispersal rates are reasonable.

Apart from the stationary patches, the model (1-3) admits various dynamic patterns such as periodic, quasi-periodic and chaotic patterns when the stationary coexistence is destroyed due to reasonable competition pressure exerted by one species on another and moderate dispersal rates of all species. Here, we have considered the third species as stronger competitor and the second species moves moderately faster than others. Chaotic pattern exists in a larger parametric region than other dynamic patterns and it leads to irregular oscillations of densities with respect to both the space and time. The irregular oscillations emerge due to continuous migration of species in neighboring patches, and continuous change in both the configuration and size of patches (Banerjee and Volpert 2016). As a result, this phenomenon prevents a species from being completely extinct by inducing its localized and temporary extinction. Figure $4 \mathrm{f}$ demonstrates that the first two species invade the third species infested region from the neighboring sites (see also Fig. 6). In the usual sense, the invasion by the first two species would have meant the successful establishment of them when few individuals of these species were introduced within a small patch of the whole domain initially occupied by the third species (Adamson and Morozov 2012; Manna and Banerjee 2018). We can call it as the short-range invasion by the first two species which is a significant outcome of our study.

Further, we have showed that the specific spatial system (25-27) does not admit any stationary Turing pattern which can supplement the studies conducted in Adamson and Morozov (2012), Petrovskii et al. (2001). Thus, limited variabilities in the rate constants favor the stationary or dynamic multi-species coexistence through selforganization (Camazine et al. 2001; Nicolis and Prigogine 1977). The formation of localized population patches is important for species persistence and this phenomenon cannot arise in the corresponding non-spatial system. This is due to the fact that individuals of weak competitors can avoid competition pressure by moving to a location with relatively low density of strong competitors. Also, the spatial mobility of a species increases the accessibility of resources to a great extent.

An individual of a species can consume available resources from surroundings due to its spatial mobility and hence, the nonlocal intra-specific competition comes into picture (Autry et al. 2018; Britton 1989; Manna et al. 2020; Merchant and Nagata 2011; Tanzy et al. 2013). Accordingly, we have investigated the effects of the nonlocal intraspecific competitions on the local spatiotemporal dynamics possessed by the spatial system (1-3) in Sect. 3. Numerical simulations for the parameter values corresponding to the first type of cyclic ordering with different values of the range of nonlocal interaction (that is, $\delta$ ) lead to the expansion of the Turing instability region (see Fig. 10). In such a way, both the Turing and Hopf-Turing domains engulf the whole $\left(a_{13}, d_{2}\right)$ parameter space for $\delta=2$ as the remaining two domains (stable and Hopf domains) almost disappear. Accordingly, appropriate value of $\delta$ can transform the oscillatory population distribution to stationary one and this phenomenon can be considered as nonlocal interaction induced stabilization of the system dynamics. Therefore, this type 
of stabilization suggests that resource consumption from surroundings strengthens the formation of stationary population patches (see Fig. 12). Also, sufficiently small value of $\delta$ retains the nature of the dynamics of the corresponding local spatial system; however, it breaks the spatial symmetry of the emerging pattern.

The above-mentioned stabilizing and symmetry-breaking capabilities of the nonlocal intra-specific competitions have been already discussed in Manna et al. (2020). Surprisingly, the system collapses as one species goes extinct for intermediate values of $\delta$ and the bio-diversity is regained for sufficiently large values of $\delta$ (see Figs. 13 and 14). As far as our knowledge goes, the nonlocality induced bio-diversity loss has not been reported earlier. Finally, we have shown that the nonlocal system corresponding to the specific model (25-27) can have stationary spatially heterogeneous patterns which are not achievable without nonlocal intra-specific competitions. Both the spatial mobility and nonlocal consumption of resources enhance the survival of multiple competing species, but the nature of patches (whether stationary or dynamic) is determined by competition strengths, dispersal rates and range of nonlocal interaction. We would like to mention that the study regarding the dynamics of a nonlocal three-species competition model is rare; however, only a few studies have been conducted to investigate the complex spatiotemporal dynamics of nonlocal two-species competition models (Apreutesei et al. 2008; Ni et al. 2018; Segal et al. 2013; Tanzy et al. 2013).

In this study, we have used the top-hat kernel to model the nonlocal intra-specific interactions as a starting point. It would be interesting to validate our observations with other forms of kernel, namely parabolic, triangular, Laplacian and Gaussian kernels (Merchant and Nagata 2011; Pal et al. 2019; Segal et al. 2013), as the nature of nonlocal interaction varies from one species to another. Nevertheless, nonlocality could arise for the inter-specific competitions (Banerjee and Volpert 2016). Therefore, it would be another compelling research prospect to explore the dynamics of the model in the presence of the nonlocal inter-specific competitions. As the movement of a species from one spatial location to another requires time in the case of long range dispersal, it would be interesting to consider spatiotemporal kernels (which depend on both the space and time) instead of spatial kernels (Britton 1990; Gourley and Britton 1996; Pal et al. 2020). Accordingly, it would be another fascinating future research prospect to study the effects of spatiotemporal kernels on resulting coexistence scenario of threespecies competition model. We will consider these open problems in a systematic fashion for our future studies.

Acknowledgements The first author gratefully acknowledges the financial support provided by Indian Institute of Technology Kanpur for pursuing his post-doctoral research. The second author was supported by the Ministry of Science and Higher Education of the Russian Federation: agreement no. 075-03-2020223/3 (FSSF-2020-0018). The work of the third author was supported by SERB grant MTR/2018/000527. The authors express their gratitude to the learned reviewers for the insightful comments and suggestions.

\section{Declarations}

Conflicts of interest The authors declare that they have no conflict of interest. 


\section{Appendix A}

Here, we present the phase portraits of the spatially averaged densities of all the three competitive species for the dynamic patterns illustrated in Fig. 4. Thus, it would be appropriate to define what we mean by the spatially averaged densities. The spatially averaged densities $\left\langle N_{j}>(t)\right.$ over an one-dimensional spatial domain $[-L, L]$ are given by

$$
<N_{j}>(t)=\frac{1}{2 L} \int_{-L}^{L} N_{j}(x, t) d x
$$

for $j=1,2,3$.

\section{References}

Adamson MW, Morozov AY (2012) Revising the role of species mobility in maintaining biodiversity in communities with cyclic competition. Bull Math Biol 74:2004-2031

Apreutesei N, Ducrot A, Volpert V (2008) Competition of species with intra-specific competition. Math Modelling Nat Phenom 3:1-27

Autry EA, Bayliss A, Volpert VA (2018) Biological control with nonlocal interactions. Math Biosci 301:129146

Baer SM, Li B, Smith HL (2006) Multiple limit cycles in the standard model of three species competition for three essential resources. J Math Biol 52:745-760

Banerjee M, Volpert V (2016) Prey-predator model with a nonlocal consumption of prey. Chaos 26:083120

Bayliss A, Volpert VA (2017) Complex predator invasion waves in a Holling-Tanner model with nonlocal prey interaction. Physica D 346:37-58

Britton NF (1989) Aggregation and the competitive exclusion principle. J Theor Biol 136:57-66

Britton NF (1990) Spatial structures and periodic travelling waves in an integro-differential reactiondiffusion population model. SIAM J Appl Math 50:1663-1688

Buss LW, Jackson JBC (1979) Competitive networks: nontransitive competitive relationships in cryptic coral reef environments. Am Nat 113:223-234

Camazine S, Deneubourg J-L, Franks NR, Sneyd J, Theraulaz G, Bonabeau E (2001) Self-organization in biological systems. Princeton University Press, New Jersey

Cangiani A, Georgoulis EH, Morozov AY, Sutton OJ (2018) Revealing new dynamical patterns in a reactiondiffusion model with cyclic competition via a novel computational framework. Proc. R. Soc. A. 474:20170608

Chi C, Hsu S, Wu L (1998) On the asymmetric May-Leonard model of three competing species. SIAM J Appl Math 58:211-226

Coste J, Peyraud J, Coullet P (1979) Asymptotic behaviors in the dynamics of competing species. SIAM J Appl Math 36:516-543

Gardini L, Lupini R, Messia MG (1989) Hopf bifurcation and transition to chaos in Lotka-Volterra equation. J Math Biol 27:259-272

Gilpin ME (1975) Limit cycles in competition communities. Am Nat 109:51-60

Gourley SA (2000) Travelling front solutions of a nonlocal Fisher equation. J Math Biol 41:272-284

Gourley SA, Britton NF (1996) A predator-prey reaction-diffusion system with nonlocal effects. J Math Biol 34:297-333

Han Y-T, Han B, Zhang L, Xu L, Li M-F, Zhang G (2011) Turing instability and wave patterns for a symmetric discrete competitive Lotka-Volterra system. WSEAS Trans Math 10:181-189

Jackson JBC, Buss L (1975) Alleopathy and spatial competition among coral reef invertebrates. Proc Nat Acad Sci USA 72:5160-5163

Kerr B, Riley MA, Feldman MW, Bohannan BJM (2002) Local dispersal promotes biodiversity in a real-life game of rock-paper-scissors. Nature 418:171-174

Kirkup BC, Riley MA (2004) Antibiotic-mediated antagonism leads to a bacterial game of rock-paperscissors in vivo. Nature 428:412-414 
Kishimoto K (1982) The diffusive Lotka-Volterra system with three species can have a stable non-constant equilibrium solution. J Math Biol 16:103-112

Li Q, Liu Z, Yuan S (2019) Cross-diffusion induced Turing instability for a competition model with saturation effect. Appl Math Comput 347:64-77

Lou Y, Ni W-M (1996) Diffusion, self-diffusion and cross-diffusion. J Diff Equ 131:79-131

Lou Y, Martínez S, Ni W-M (2000) On $3 \times 3$ Lotka-Volterra competition systems with cross-diffusion. Discret Contin Dyn Syst 6:175-190

Manna K, Banerjee M (2018) Stationary, non-stationary and invasive patterns for a prey-predator system with additive Allee effect in prey growth. Ecol Complex 36:206-217

Manna K, Volpert V, Banerjee M (2020) Dynamics of a diffusive two-prey-one-predator model with nonlocal intra-specific competition for both the prey species. Mathematics 8:101

Martínez S (2003) The effect of diffusion for the multispecies Lotka-Volterra competition model. Nonlinear Anal Real World Appl 4:409-436

May RM, Leonard WJ (1975) Nonlinear aspects of competition between three species. SIAM J Appl Math 29:243-253

Merchant SM, Nagata W (2011) Instabilities and spatiotemporal patterns behind predator invasions with nonlocal prey competition. Theor Popul Biol 80:289-297

Merchant SM, Nagata W (2015) Selection and stability of wave trains behind predator invasions in a model with non-local prey competition. IMA J Appl Math 80:1155-1177

Merino S (1996) Cyclic competition of three species in the time periodic and diffusive case. J Math Biol 34:789-809

Moen J (1989) Diffuse competition: a diffuse concept. Oikos 54:260-263

Mukherjee N, Ghorai S, Banerjee M (2018) Effects of density dependent cross-diffusion on the chaotic patterns in a ratio-dependent prey-predator model. Ecol Complex 36:276-289

Murray JD (1989) Mathematical biology. Springer, Heidelberg

Ni W, Shi J, Wang M (2018) Global stability and pattern formation in a nonlocal diffusive Lotka-Volterra competition model. J Differ Equ 264:6891-6932

Nicolis G, Prigogine I (1977) Self-organization in nonequilibrium systems: from dissipative structures to order through fluctuations. Wiley, New York

Pal S, Ghorai S, Banerjee M (2018) Analysis of a prey-predator model with non-local interaction in the prey population. Bull Math Biol 80:906-925

Pal S, Ghorai S, Banerjee M (2019) Effect of kernels on spatio-temporal patterns of a non-local prey-predator model. Math Biosci 310:96-107

Pal S, Banerjee M, Volpert V (2020) Spatio-temporal Bazykin's model with space-time nonlocality. Math Biosci Eng 17:4801-4824

Paquin CE, Adams J (1983) Relative fitness can decrease in evolving asexual populations of S. cerevisial. Nature 306:368-371

Pascual M (1993) Diffusion-induced chaos in a spatial predator-prey system. Proceed R Soc B Biol Sci 251:1-7

Petrovskii S, Kawasaki K, Takasu F, Shigesada N (2001) Diffusive waves, dynamical stabilization and spatio-temporal chaos in a community of three competitive species. Jpn J Ind Appl Math 18:459-481

Schuster P, Sigmund K, Wolff R (1979) On $\omega$-limits for competition between three species. SIAM J Appl Math 37:49-54

Segal BL, Volpert VA, Bayliss A (2013) Pattern formation in a model of competing populations with nonlocal interactions. Physica D 253:12-22

Smale S (1976) On the differential equations of species in competition. J Math Biol 3:5-7

Tanzy MC, Volpert VA, Bayliss A, Nehrkorn ME (2013) Stability and pattern formation for competing populations with asymmetric nonlocal coupling. Math Biosci 246:14-26

Turing AM (1952) The chemical basis of morphogenesis. Philos Trans R Soc London B Biol Sci 237:37-72

van Voorn GAK, Hemerik L, Boer MP, Kooi BW (2007) Heteroclinic orbits indicate overexploitation in predator-prey systems with a strong Allee effect. Math Biosci 209:451-469

Wolf A, Swift JB, Swinney HL, Vastano JA (1985) Determining Lyapunov exponents from a time series. Physica D 16:285-317

Publisher's Note Springer Nature remains neutral with regard to jurisdictional claims in published maps and institutional affiliations. 


\section{Affiliations}

\section{Kalyan Manna ${ }^{1} \cdot$ Vitaly Volpert $^{2,3,4} \cdot$ Malay Banerjee $^{1}$}

$凶 \quad$ Malay Banerjee

malayb@iitk.ac.in

Kalyan Manna

kalyan274667@gmail.com

Vitaly Volpert

volpert@math.univ-lyon1.fr

1 Department of Mathematics and Statistics, Indian Institute of Technology Kanpur, Kanpur 208016, Uttar Pradesh, India

2 Institut Camille Jordan, UMR 5208 CNRS, University Lyon 1, 69622 Villeurbanne, France

3 INRIA, Team Dracula, INRIA Lyon La Doua, 69603 Villeurbanne, France

4 Peoples' Friendship University of Russia (RUDN University), 6 Miklukho-Maklaya St, Moscow 117198, Russia 